\title{
Simulation Study of Autonomous Vehicles' Effect on Traffic Flow Characteristics including Autonomous Buses
}

\author{
Tanveer Muhammad $\left(\mathbb{D},{ }^{1}\right.$ Faizan Ahmad Kashmiri $\left(\mathbb{D},{ }^{2}\right.$ Hassan Naeem, ${ }^{3}$ Xin Qi $\left(\mathbb{D},{ }^{1}\right.$ \\ Hsu Chia-Chun, ${ }^{1}$ and Huapu Lu (i) ${ }^{1}$ \\ ${ }^{1}$ Department of Civil Engineering, Tsinghua University, Beijing 100084, China \\ ${ }^{2}$ Department of Civil and Environmental Engineering, The Hong Kong University of Science and Technology, Hong Kong, China \\ ${ }^{3}$ Lahore Transport Company (LTC), Lahore, Pakistan
}

Correspondence should be addressed to Huapu Lu; luhp@mail.tsinghua.edu.cn

Received 19 July 2019; Revised 24 December 2019; Accepted 29 January 2020; Published 4 July 2020

Academic Editor: Stefano de Luca

Copyright (c) 2020 Tanveer Muhammad et al. This is an open access article distributed under the Creative Commons Attribution License, which permits unrestricted use, distribution, and reproduction in any medium, provided the original work is properly cited.

\begin{abstract}
Autonomous vehicles are expected to revolutionize the transportation industry. The goal of this research is to study the heterogeneity in traffic flow dynamics by comparing different penetration rates of four different types of vehicles: autonomous cars $(\mathrm{AC})$, autonomous buses $(\mathrm{AB})$, manual cars $(\mathrm{MC})$, and manual buses (MB). For the purpose of this research, a modified cellular automata (CA) model is developed in order to analyze the effect of heterogeneous vehicles (manual and autonomous). Previously, studies have focused on manual and autonomous cars, but we believe a gap in perception and analysis of mixed traffic still exists, as inclusion of other modes of autonomous vehicle research is very limited. Therefore, we have explicitly examined the effect of the $\mathrm{AB}$ on overall traffic flow. Moreover, two types of lane changing behavior (aggressive lane changing and polite lane changing) were also integrated into the model. Multiple scenarios through different compositions of vehicles were simulated. As per the results, if $\mathrm{AB}$ is employed concurrently with $\mathrm{AC}$, there will be a significant improvement in traffic flow and road capacity, as equally more passengers can be accommodated in $\mathrm{AB}$ as $\mathrm{AC}$ is also anticipated to be used in carpooling. Secondly, when the vehicles change the lanes aggressively, there is a substantial growth in the flow rate and capacity of the network. Polite lane change does not significantly affect the flow rate.
\end{abstract}

\section{Introduction}

The increasing level of urbanization throughout major cities across the globe is leading to numerous problems, one of which is traffic congestion. The main cause of congestion is the unbalanced ratio of supply and demand. The lengthening of journey time due to congestion increases fuel consumption, deteriorates the environment, and brings about traffic accidents [1]. In 2018, the World Health Organization reported that 1.35 million people die per year due to traffic accidents. The empirical study found out that the major causes of road accidents are driver's conduct, environmental conditions, roads, and vehicles' technical conditions [2].

Day by day, cities have gone through numerous transformations regarding urban transportation systems. In order to realize smooth traffic flow, guarantee traffic safety, and reduce travel time, pollution, and congestion, wireless communication and sensing technologies have swiftly and progressively been integrated within the past two decades. In the coming years, transportation industries, scholars, and software companies will continue their efforts to implement vehicle-based innovations in order to improve transportation systems. Autonomous vehicles are one of those revolutionary trends, namely, due to their advanced control intelligence, pattern recognition, and sensing skills. Owing to the universal wireless communication technologies, communications between vehicles, infrastructure, and pedestrian are possible. Autonomous vehicles maneuver via road networks and drivers do not need to be supervisors or decision-makers. It is expected that autonomous vehicles 
will change the driving environment and will come into the market by 2020 . Therefore, in the next two decades, we will need to figure out the effect of mixed traffic flow on the urban road. These different types of vehicles have a different driving framework. Autonomous vehicles have short headway and reduce reaction time as compared to humandriven vehicles [3].

As autonomous vehicles will affect the traffic flow characteristics at both macro and micro levels, we need to study the effects of autonomous vehicles on traffic flow. Previous simulation studies have indicated that the capacity prominently increases by reducing the headway and reaction time [4]. Traffic systems have the potential to be fully autonomous in the future. Although multiple studies have been conducted on mixed traffic, including autonomous cars and manual cars in the past, in this paper, we attempt to analyze the traffic flow characteristics with mixed autonomous vehicles (including cars and buses) and conventional vehicles (including bus and car) with a different penetration rate of autonomous vehicles. An autonomous bus is involved in this research to investigate its impact on traffic flow characteristics as it has the potential to change the existing transportation systems. We will explore the results in the form of traffic flow fundamentals, that is, flow rate, speed, and density. Lane changing is one of the essential parameters for traffic flow. We will also consider the lane change behavior under the circumstances of mixed autonomous vehicles and conventional vehicles.

The rest of the paper is designed as follows. Section 2 focuses on the literature review of mixed traffic flow (including automated vehicle and conventional vehicles) characteristics and lane change behavior under mixed traffic conditions; describes the introduction of the cellular automata (CA) model structure, Nagel-Schreckenberg model, and improvement in the CA model structure according to an ideal situation in mixed transportation systems; and describes the series of simulations of the different penetration rates of autonomous vehicles and manual vehicles. Section 3 provides a discussion based on simulation results. Section 4 presents the conclusion and gives further suggestions for future research.

\section{Literature Review}

The impact of autonomous vehicles (AVs) on the current transport system, such as traffic safety, congestion, travel behavior, parking, and ownership of vehicles, has been much discussed in the past half decade. Fagnant and Kockelman summarized that the AVs represent a potentially groundbreaking but a positive change to our transport system in terms of vehicle safety, travel behavior, and congestion [5]. In particular, AVs have a much quicker reaction time and short headway; therefore, the traffic capacity of roads with AVs is therefore expected to increase [6-9]. AVs can also help reduce fuel and emissions. At the beginning of deployment, since the penetration rate of AVs will be low, the overall traffic characteristics of AVs in accordance with manual vehicles will be complicated and difficult to comprehend $[10,11]$.
Appropriate modeling approaches are required at all levels (micro and macro), which could allow the evolving dynamics and capacities of mixed traffic flow to be properly reflected.

What we are mainly concerned with in this research is the impact of an autonomous vehicle (including the autonomous car $(\mathrm{AC})$ and autonomous bus $(\mathrm{AB})$ ) on the road capacity. We will simulate the heterogeneous traffic flow of autonomous vehicles and manual vehicles and subsequently analyze the influence of $\mathrm{AC}$ and $\mathrm{AB}$ on road capacity and traffic flow.

The influence of AVs on road capacity represents a huge area for debates. Tientrakool et al. (2011) have analyzed the capacity under mixed traffic flow conditions and an increase in highway capacity through the introduction of autonomous vehicles [12]. Van Arem et al. in their study have shown that the introduction of AVs can reduce capacity even when the share of them is low [13]. Previous studies on autonomous vehicles have generally assumed the autonomous vehicle to be of single mode, namely, car. In real-time scenarios, there will be different modes of autonomous vehicles running in parallel with manual vehicles. The focus of the study is the analysis of mixed traffic flow of vehicles (autonomous car, autonomous bus, manual car, and manual bus) running on multiple lanes. Moreover, our additional goal is to examine the impact of autonomous vehicles on the overall network when fused with different lane change (LC) behavior.

Lane changing behavior is one of the primary characteristics of the traffic. Lane changes have a negative influence on road safety and traffic flow [14] and frequent lane changing behavior is one of the reasons for congestion and/ or accidents $[15,16]$. Gipps was the first person to introduce the lane changing model for traffic [17]. While several other models were developed based on Gipps's model, Hidas developed a better-quality modeling framework for urban roads and highways to capture LC's vehicle interaction, which was explicitly classified into three categories: free, cooperative, and forced lane change based on the video recording observations [14]. Ahmed et al. developed the lane change decision model by implementing utility theory to lane change behavior [18]. Kesting et al. developed MOBIL (Minimizing Overall Braking Induced by Lane changes) model for simplifying and modeling lane change decisions [19]. This MOBIL model is similar to the Gipps model; the only difference is that rules in MOBIL are accelerationbased. Other scientists have further studied intelligent lane changing models based on MOBIL [20]. Tawari et al. [21] and Hou et al. [22] investigated the concept of safety: whether it is safe for a vehicle to implement a lane changing maneuver depending upon the traffic situation. Scheduling of connected autonomous vehicles in terms of lane changing was considered by $\mathrm{Hu}$ et al. [23] and Awal et al. [24]. Awal et al. [25] proposed an optimal merging strategy for communication and sensor-enabled vehicles. Desiraju et al. [26] developed an algorithm to reduce the disruption of the traffic flow of autonomous vehicles during lane changing maneuvers. It is expected that the development of autonomous vehicles offers great opportunities for the future. 
Since the AVs are not available in the open market, we can analyze their influence only through simulation studies. For this purpose, we can choose either cellular automata or VISSIM and AIMSUN. The major concern in our study is to investigate the impact of different modes of autonomous vehicles in mixed traffic flow and assess them through traffic fundamental diagrams. For this particular reason, a mesoscopic traffic model such as CA or a microscopic traffic model such as VISSIM or AIMSUN should be adopted. CA model can easily simulate nonlinear complex traffic flow dynamics and provide elasticity by simulating multifaceted and sophisticated real-time traffic scenarios as suggested in previous studies. Furthermore, the dynamics of traffic flow can be simulated by incorporating different elements in one time. Another major goal of this study is to assess the impact of different lane changing behaviors in different simulations, which are easy to simulate in CA model due to its inherent simplicity. In view of the ability of CA to model complex nonlinear traffic flow dynamics, CA has been designated as the most appropriate tool for the simulation of real traffic flows due to their efficient and speedy performance in computer simulations. The CA model can reproduce accurate traffic events such as the transition from free flow to congestion, lane inversion, and platoon formation. The CA model is discrete in time and space. Depending on different requirements, we can define unit time and unit length of the cell. The CA model is selected for simulation in this study, based on the aforementioned advantages.

Nagel and Schreckenberg introduced the cellular automata model for traffic simulation known as the NaSch model [27]. Later, several model extensions of NaSch model were developed, such as Fukui-Ishibashi (FI) model, VE model, VDR model, and TT model [28-33]. Two-lane models were proposed for simulating the real traffic. Rickert et al. explored a simple two-lane cellular automata (CA) model based on Nagel and Schreckenberg's proposed single-lane CA [34].

Different CA models were developed over time to investigate the impact of different lane change behaviors [35-39]. Gao et al. developed a traffic flow CA model in the context of Kerner's three-phase traffic theory [40]. Chen et al. introduced a new CA model in which the randomization effect is enriched by reducing the time headway [41]. Tonguz et al. developed a new CA approach to build a model for urban traffic mobility [42]. Das proposed a CA model, allowing vehicles to move at low speed during congestion [43]. Previous studies have also developed CA models that simulate multiclass traffic flows [44-46]. Recently, Liu et al. explored the impact of autonomous vehicles and their penetration rate on heterogeneous traffic flow characteristics in an improved CA model $[47,48]$. Their study mostly focused on autonomous cars and manual cars. In this study, we have improved their CA model by incorporating new modes of manual and autonomous vehicles such as manual car, manual bus, autonomous car, and a new type of autonomous bus. Furthermore, we have also developed different lane changing policies for different modes of vehicles. This kind of model is essential to study the impact of different types of autonomous vehicles on overall traffic flow dynamics.
Thus, the CA model is helpful in simulating real-time traffic scenarios with different vehicular characteristics, which are the basis of this study as both manual and autonomous vehicles will have distinct attributes.

2.1. Model. In this new developed CA model, we simulate the behavior of different types of vehicles. All the parameters of the model are described in Table 1. We have four types of vehicles such as the autonomous car (AC), manual car (MC), autonomous bus $(\mathrm{AB})$, and manual bus $(\mathrm{MB})$. Regarding $\mathrm{AC}$, the lengths of these cars do not exceed 5 meters and their size is equal to the size of each cell in the model. The main differences between $\mathrm{AC}$ and $\mathrm{MC}$ are a delay for reaction and policy for a lane change. AC can follow both aggressive lane change (ALC) and polite lane change (PLC).

2.1.1. MC. Manual cars (MC) are similar to AC but they usually have more reaction delay than $\mathrm{AC}$ and they follow ALC policies for lane changing. Another feature of this kind of car is a random brake. Since people are not able to keep the speed constant, this kind of cars usually does take some brake randomly. The time step is one second; therefore, in each time step, some of MC cars brake and decrease their speed randomly. All the updating rules for cars are explained in Table 2. The minimum distance for the manual car $\left(D_{n, \mathrm{mc}}\right)$ from the preceding vehicle is 2 cells.

2.1.2. $A B$. Autonomous buses $(\mathrm{AB})$ are the third type of vehicle in our model. They have more length than $\mathrm{AC} / \mathrm{MC}$ cars and, usually, the length is 2 times longer than $\mathrm{AC} / \mathrm{MC}$ and they occupy 2 cells of a line. This kind of vehicle also has different reaction delays and lane changing options. The minimum distance for an autonomous bus $\left(D_{n, \mathrm{ab}}\right)$ from the preceding vehicle is 1 cell.

2.1.3. $M B$. Manual bus (MB) is the fourth type of vehicle. The manual bus has slightly different features such as delay time and random brake system. In each step, we update our simulation by following the Nagel-Schreckenberg Model. There is a significant difference in the previous model as we are simulating different kinds of vehicles so we have assigned the different headway for each vehicle. The minimum distance for manual car $\left(D_{n, \mathrm{mb}}\right)$ from the preceding vehicle is 3 cells. All the updating rules for the bus are explained in Table 3 and in Table 4 all the fundamental parameters of the model are explained.

Figure 1 represents the schematic diagram, illustrating the various parameters involved in the model.

2.2. Lane Changing. We have considered lane policies, that is, aggressive and polite policies. Autonomous vehicles follow both policies, but manual cars follow only aggressive policies. Any vehicle, either autonomous or manual, is assumed to comply with a basic rule that lane change can only be carried out at the least cost of reducing the speed of neighboring vehicles; that is, a vehicle would only change its 
TABle 1: Parameters in the model.

\begin{tabular}{|c|c|}
\hline Parameters & Description \\
\hline$D_{n, \mathrm{ac}}(\mathrm{m})$ & The distance of the $n$ autonomous car from the preceding vehicle \\
\hline$D_{n, m c}(\mathrm{~m})$ & The distance of the $n$ manual car from the preceding vehicle \\
\hline$D_{n, \mathrm{ab}}(\mathrm{m})$ & The distance of $n$ autonomous bus from the preceding vehicle \\
\hline$D_{n, \mathrm{mb}}(\mathrm{m})$ & The distance of the $n$ manual bus from the preceding vehicle \\
\hline$D_{n, \mathrm{ac}, l}(\mathrm{~m})$ & Left side front distance of the $n$ autonomous car in the target line \\
\hline$D_{n, \mathrm{mc}, l}(\mathrm{~m})$ & Left side front distance of the $n$ manual car in the target line \\
\hline$D_{n, \mathrm{ab}, l}(\mathrm{~m})$ & Left side front distance of the $n$ autonomous bus in the target line \\
\hline$D_{n, \mathrm{mb}, l}(\mathrm{~m})$ & Left side front distance of the $n$ manual bus in the target line \\
\hline$D_{n, \text { ac, }, \text { back }}(\mathrm{m})$ & Left side back distance of the $n$ autonomous car in the target line \\
\hline$D_{n, \mathrm{mc}, l, \text { back }}(\mathrm{m})$ & Left side back distance of $n$ manual car in the target line \\
\hline$D_{n, \mathrm{mb}, l, \text { back }}(\mathrm{m})$ & Left side back distance of the $n$ manual bus in the target line \\
\hline$D_{n, \text { ab }, l, \text { back }}(\mathrm{m})$ & Left side back distance of the $n$ autonomous bus in the target line \\
\hline$D_{n, \mathrm{ac}, r}(\mathrm{~m})$ & Right side front distance of the $n$ autonomous car in the target line \\
\hline$D_{n, \mathrm{mc}, r}(\mathrm{~m})$ & Right side front distance of the $n$ manual car in the target line \\
\hline$D_{n, \mathrm{ab}, r}^{n, 1 \mathrm{~m})}$ & Right side front distance of the $n$ autonomous bus in the target line \\
\hline$D_{n, \mathrm{mb}, r}(\mathrm{~m})$ & Right side front distance of the $n$ manual bus in the target line \\
\hline$D_{n, \mathrm{ac}, r, \mathrm{back}}(\mathrm{m})$ & Right side back distance of the $n$ autonomous car in the target line \\
\hline$D_{n, \mathrm{mc}, r, \text { back }}(\mathrm{m})$ & Right side back distance of $n$ manual car in the target line \\
\hline$D_{n, \mathrm{mb}, l, \text { back }}(\mathrm{m})$ & Right side back distance of the $n$ manual bus in the target line \\
\hline$D_{n, \mathrm{ab}, l, \mathrm{back}}(\mathrm{m})$ & Right side back distance of the $n$ autonomous bus in the target line \\
\hline$D_{0}(\mathrm{~m})$ & The size of each cell \\
\hline$v_{n, \mathrm{ac}}(t)$ & The current speed of an $n$ autonomous car \\
\hline$v_{n, \mathrm{mc}}(t)$ & The current speed of an $n$ manual car \\
\hline$v_{n, \mathrm{ab}}(t)$ & The current speed of an $n$ autonomous bus \\
\hline$v_{n, \mathrm{ab}}(t)$ & The current speed of an $n$ manual bus \\
\hline$v_{\mathrm{ac}, \max }$ & The maximum speed of an $n$ autonomous car \\
\hline$v_{\mathrm{mc}, \max }$ & The maximum speed of an $n$ manual car \\
\hline$v_{\mathrm{ab}, \max }$ & The maximum speed of an $n$ autonomous bus \\
\hline$v_{\mathrm{mb}, \max }$ & The maximum speed of an $n$ manual bus \\
\hline$v_{n, r, \text { back }}(t)$ & The speed of back vehicle on the right side in target line \\
\hline$v_{n, l, \text { back }}(t)$ & The speed of back vehicle on the left side in target line \\
\hline
\end{tabular}

TABLE 2: Rules for updating the position of different cars in the model.

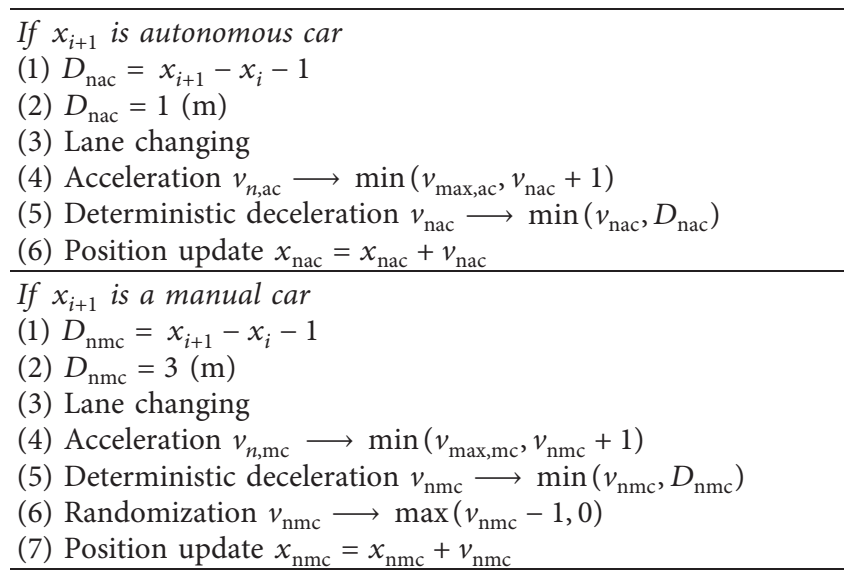

lane if its neighboring vehicles on the target lane do not have to slow down too much. Furthermore, the change of lane is acceptable in either direction. In particular, two sets of lane changing rules, polite lane changing (PLC) and aggressive lane changing (ALC), are considered. Depending on the situation, an autonomous vehicle can follow either a PLC or ALC, while a manual vehicle can follow the ALC rule only. We have modified the rules according to different types of
TABle 3: Rules for updating the position of different buses in the model.

If $x_{i+1}$ is autonomous bus

(1) $D_{\text {nab }}=x_{i+1}-x_{i}-2$

(2) $D_{\text {nab }}=1(\mathrm{~m})$

(3) Lane changing

(4) Acceleration $v_{n, \mathrm{ab}} \longrightarrow \min \left(v_{\mathrm{max}, \mathrm{ab}}, v_{\mathrm{nab}}+1\right)$

(5) Deterministic deceleration $v_{\text {nab }} \longrightarrow \min \left(v_{\text {nab }}, D_{\text {nab }}\right)$

(6) Position update $x_{\text {nab }}=x_{\text {nab }}+v_{\text {nab }}$

If $x_{i+1}$ is manual bus

(1) $D_{\mathrm{nmb}}=x_{i+1}-x_{i}-2$

(2) $D_{\mathrm{nmb}}=4(\mathrm{~m})$

(3) Lane changing

(4) Acceleration $v_{n, \mathrm{mb}} \longrightarrow \min \left(v_{\text {max }, \mathrm{mb}}, v_{\mathrm{nac}}+1\right)$

(5) Deterministic deceleration $v_{\mathrm{nmb}} \longrightarrow \min \left(v_{\mathrm{nmb}}, D_{\mathrm{nmb}}\right)$

(6) Randomization $v_{\mathrm{nmb}} \longrightarrow \max \left(v_{\mathrm{nmb}}-1,0\right)$

(7) Position update $x_{\mathrm{nmb}}=x_{\mathrm{nmb}}+v_{\mathrm{nmb}}$

vehicles. There are three lanes in the mode. AC and MC can utilize all the lanes. However, buses are restricted to operate on the second and third lane.

For the right lane changing case, same nomenclature applies as the left lane. However, vehicles will prefer the left lane change rules if the left lane change rules meet the criterion of safety. The vehicle also checks the lane change possibility on the right: if the criterion of safety is not met, vehicle $n$ remains in 
TABLE 4: Fundamental parameters of model.

Size of each vehicle

1 cell $=5 \mathrm{~m}$

$\mathrm{AC}=1$ cell

$\mathrm{MC}=1$ cell

$\mathrm{AB}=2$ cells

$\mathrm{MB}=2$ cells

Minimum safe distance for each vehicle

$\mathrm{AC}=1$ cell

$\mathrm{MC}=2$ cells

$\mathrm{AB}=1$ cell

$\mathrm{MB}=3$ cells

Lane change

AC front and back $=2$ cells

MC front and back $=3$ cells

$\mathrm{AB}$ front and back $=2$ cells

MB front and back $=4$ cells
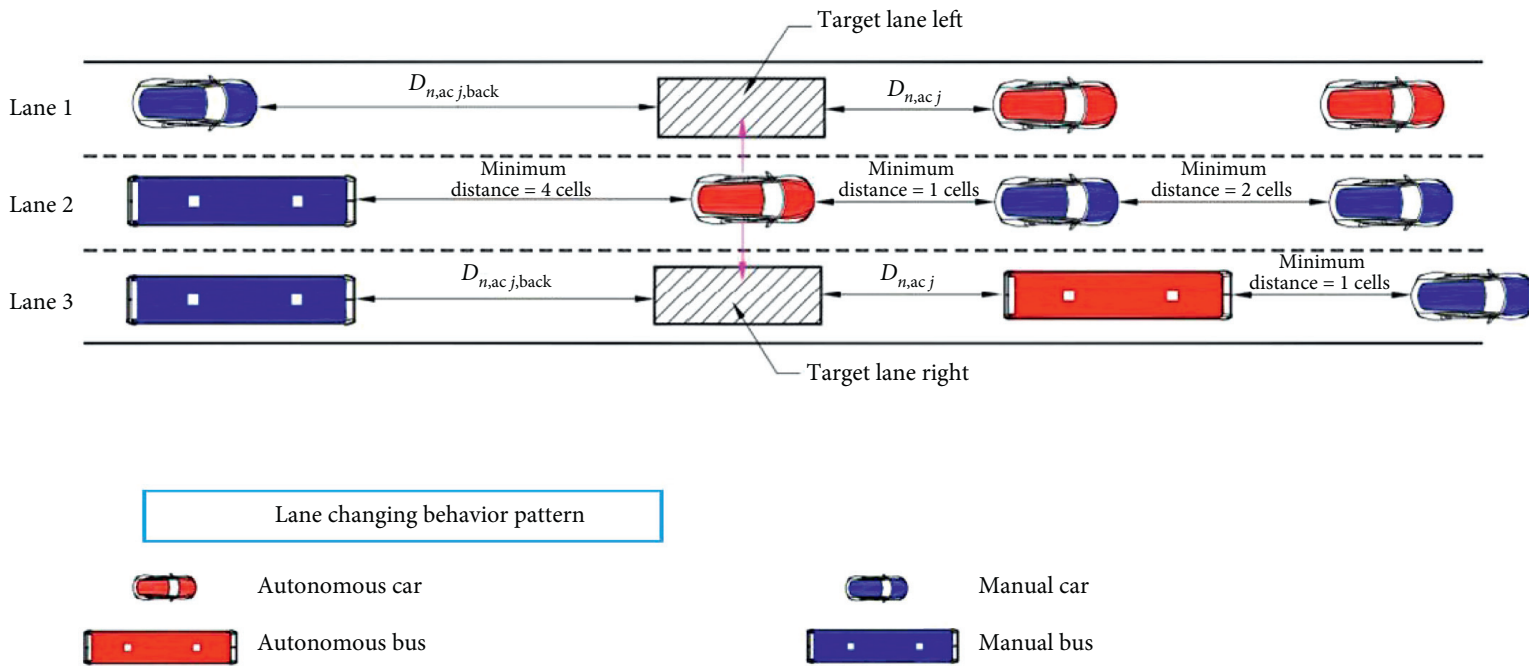

FIGURE 1: Schematic diagram of model.

the current lane. If the left and right rules are met, the vehicle $n$ may change the lane with a PC probability. This parameter is introduced to reflect the individuality of vehicles in lane changes (e.g., some vehicles may not choose to do so even under the same conditions that favor lane change).

Additionally, for lane change, in order to shift to target lane, the minimum distance for front (preceding vehicle) and back (following vehicle) for the $\mathrm{AB}$ and $\mathrm{AC}$ is 2 cells, where, for $\mathrm{MC}$, the minimum front and back distance is 3 cells and, for $\mathrm{MB}$, the minimum front and back distance is 4 cells.

2.3. Polite Policy. The fundamental hypothesis with regard to PLC is that drivers are careful when trying to change lanes; that is, their lane change maneuvers should not interfere with the movement of neighboring vehicles on the adjacent lanes. This is in line with the MOBIL model with a polity parameter of 1 . The PLC rules support a more altruistic behavior rather than assuming self-centered behavior in most of lane changing models. In general, two aspects should be taken into account during the study of lane change: incentive and safety. Only if certain criteria related to both aspects are fulfilled, vehicles will change routes. In order to set incentive and safety criteria for PLC, we refer to the STCA model. The PLC rules are shown in Table 5.

2.4. Aggressive Policy. ALC characterizes a set of lane changing rules that are more practical than PLC, particularly for manual vehicles. Report on the lane changing behavior of manual vehicles shows that slower preceding vehicles would lead the following drivers to consider overtaking in many situations [49, 50].
Table 5: Polite lane change rules.

For the autonomous car

Left lane

(i) $D_{\text {nac }}<\min \left(v_{\text {nac }}(t)+1, v_{\text {max,ac }}\right)$

(ii) $D_{\text {nac }, l}>D_{\text {nac }}$

(iii) $D_{n, l, \text { back }}>v_{\text {max,ac }}$

Right lane

(iv) $D_{\text {nac }}<\min \left(v_{\text {nac }}(t)+1, v_{\text {max,ac }}\right)$

(v) $D_{\text {nac, } r}>D_{\text {nac }}$

(vi) $D_{n, r, \text { back }}>v_{\text {max }, \text { ac }}$

For the autonomous bus

Left lane:

(vii) $D_{\text {nab }}<\min \left(v_{\text {nab }}(t)+1, v_{\text {max }, \mathrm{ab}}\right)$

(viii) $D_{n, l}>D_{\text {nab }}$

(ix) $D_{n, l, \text { back }}>v_{\text {max,ab }}$

Right lane

(x) $D_{\text {nab }}<\min \left(v_{\text {nab }}(t)+1, v_{\text {max,ab }}\right)$

(xi) $D_{n, r}>D_{\text {nab }}$

(xii) $D_{n, r, \text { back }}>v_{\text {max,ab }}$

Furthermore, 95\% of drivers would only change lanes if the rear distance on the target lane exceeds the 3-cell length (15 meters) and their speed exceeds those on the destination lane [51]. As inspired by if vehicle $n$ is a manual vehicle, $D_{0}$ is set to a length of 3 cells; otherwise, $D_{0}$ is set to a length of 2 cells to represent the different accuracy of the autonomous and manual driving behavior of the vehicles. The ALC rules are shown in Table 6 .

The deceleration rule means that the current speed of a vehicle is maintained unless it has to slow down to adapt to its current front spacing. The randomization policy will 
TABle 6: Aggressive lane change rules.

For the autonomous car

For left side

$D_{0}(\mathrm{~m})=$ cell size

$D_{0}(\mathrm{~m})=$ cell size

(i) $D_{n, l} \geq 2 * D_{0}$

(ii) $D_{n, l, \text { back }} \geq 2 * D_{0}$

(iii) $v_{\text {nac }}(t) \geq v_{n, l, \text { back }}(t)$

For right side

(iv) $D_{n, r} \geq 2 * D_{0}$

(v) $D_{n, r, \text { back }} \geq 2 * D_{0}$

(vi) $v_{\text {nac }}(t) \geq v_{n, r, \text { back }}(t)$

For the autonomous bus

For left side

$D_{0}(\mathrm{~m})=$ cell size

$D_{0}(\mathrm{~m})=$ cell size

(i) $D_{n, l} \geq 2 * D_{0}$

(ii) $D_{n, l, \text { back }} \geq 2 * D_{0}$

(iii) $v_{\text {nab }}(t) \geq v_{n, \text {,back }}(\mathbf{t})$

For right side

(iv) $D_{n, r} \geq 2 * D_{0}$

(v) $D_{n, r, \text { back }} \geq 2 * D_{0}$

(vi) $v_{\text {nab }}(t) \geq v_{n, r \text {,back }}(t)$

For the manual car

For left side

(i) $D_{n, l} \geq 3 * D_{0}$

(ii) $D_{n, l, \text { back }} \geq 3 * D_{0}$

(iii) $v_{\text {nmc }}(t) \geq v_{n, l, \text { back }}(t)$

For right side

(iv) $D_{n, r, \text { back }} \geq 3 * D_{0}$

(v) $D_{n, l} \geq 3 * D_{0}$

(vi) $v_{\text {nmc }}(t) \geq v_{n, r \text {,back }}(t)$

For the manual bus

For left side

(i) $D_{n, l} \geq 4 * D_{0}$

(ii) $D_{n, l \text {,back }} \geq 4 * D_{0}$

(iii) $v_{\text {nmb }}(t) \geq v_{n, l \text {,back }}(t)$

For right side

(iv) $D_{n, r} \geq 4 * D_{0}$

(v) $D_{n, r, \text { back }} \geq 4 * D_{0}$

(vi) $v_{\mathrm{nmb}}(t) \geq v_{n, r, \text { back }}(t)$

apply to manual vehicles with a $0<p<1$ probability. This implies that a percentage of drivers cannot keep the current speed constantly and can randomly slow their vehicles down. The position-updating rule states that vehicles update their locations at current speeds. This rule is derived from the NaSch single-lane model.

2.5. Simulation Implementation. For implementation, we used Matlab programming language. Our implementation is fully object-oriented. We have a main class that is "car class." The class has several attributes, including ID, position, speed, Max_speed, Line_max_speed, delay, type, length, line, back_space, and front_space. Each vehicle is an object of the class car and, for each vehicle, we store all of their respective parameters. In the CA model, the road segment is divided into cells. At each time step, each cell has two states occupied by either vehicle or blank. The maximum speed of the road is defined as $80 \mathrm{~km} / \mathrm{h}$. The number of lanes is 3 and road length is $10 \mathrm{~km}$. At the start of the simulation, the vehicles are randomly distributed at different cells with each vehicle size equivalent to designated number of cells as mentioned before. Furthermore, the initial speed of vehicles is set randomly. After each fixed time step, number of vehicles increases by adding vehicles again randomly in empty cells. This whole process is done again and again until the decided time period for simulation is completed.

Based on the parameters that the user set, we generate the vehicles and their features in the simulation. We have run the simulation at a different value of PLC, ALC, and probability of random brake. The simulation was run 1000 time steps for each scenario. The average speed, density, and flow rate are determined by the following formula:

$$
\begin{aligned}
& \bar{v}=\frac{1}{T} \sum_{t=t_{0}}^{T+t_{0}-1} \frac{1}{N} \sum_{n=1}^{N} v_{n}(t), \\
& k=\frac{N}{3 L}, \\
& q=k \cdot \bar{v} .
\end{aligned}
$$

\section{Results and Discussions}

The results in this paper have been discussed by comparing different scenarios, that is, different compositions of traffic. All of the results are based on the simulation model that is explained quite comprehensively in the previous section. By dividing the flow rate into the free flow phase and congested flow phase, it is easy to comprehend the significance of autonomous vehicles (cars and buses) in the model.

All the following results mentioned from Figures 2-10 delineate the differences in fundamental relations of traffic flow characteristics (speed, density, and flow rate) by comparing different penetration rates of autonomous cars $(\mathrm{AC})$, autonomous buses (AB), manual cars (MC), and manual buses (MB). Difference scenarios from 1 to 5 have been formulated based upon different compositions. In these scenarios, the values of ALC and PLC have been fixed to 0.5 to deeply understand the concept of heterogeneity in traffic and its impact through various arrangements.

In scenarios 6 to 8, different values of ALC and PLC have been incorporated in the simulation model by fixing the composition of vehicles to understand the effects of lane changing behaviors in similar configurations.

The details of these scenarios are mentioned in Table 7.

3.1. Scenario 1. Figure 2 shows the results of autonomous cars (100\% penetration) and manual cars ( $100 \%$ composition) on the same road network. The major trends off all the graphs are as expected and they perfectly align with the preestablished conventions of the discussed parameters, that is, speed, density, and flow rate. AC outperforms MC in every parameter. All of these results are generally consistent with the previous understandings primarily due to less reaction time delays from AC. The 


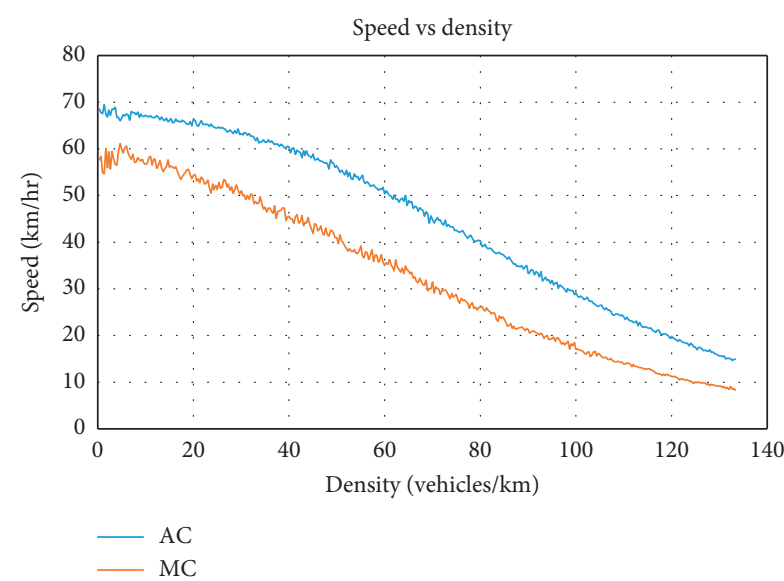

(a)

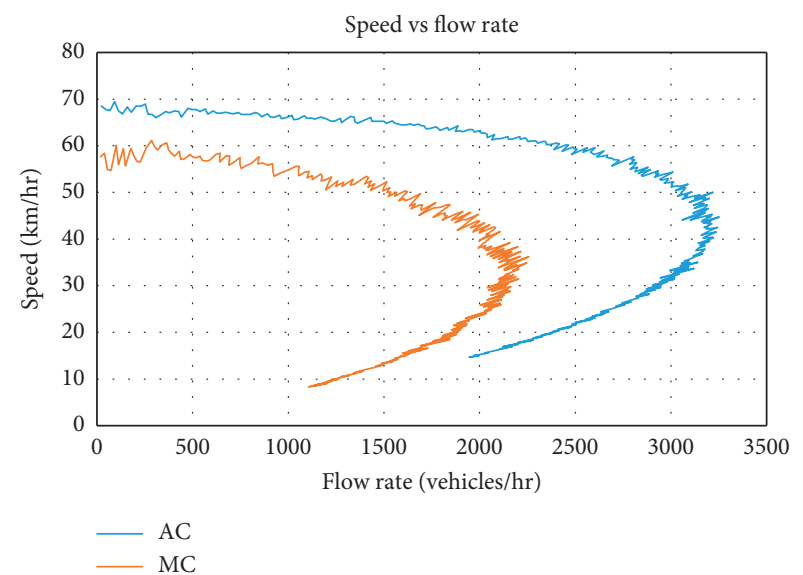

(b)

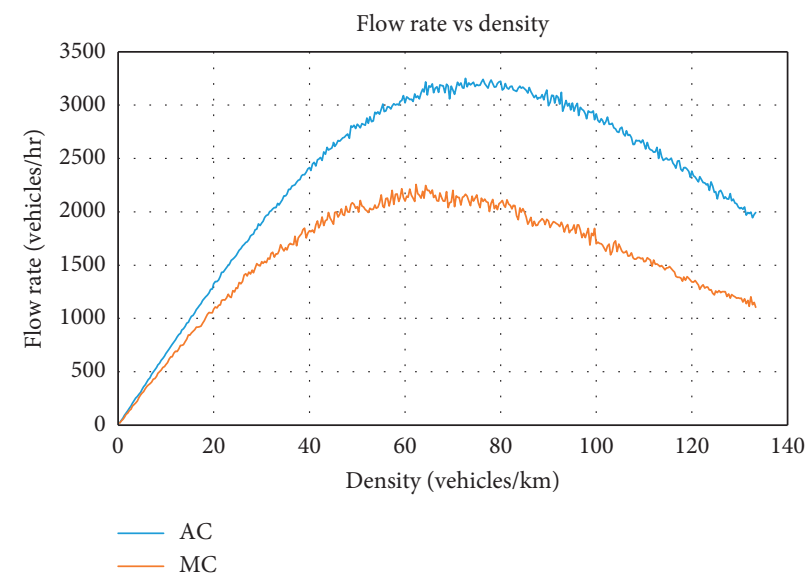

(c)

Figure 2: Traffic flow fundamental diagrams for $100 \% \mathrm{AC}$ and MC.

maximum speed for MC at $100 \%$ penetration is $60.61 \mathrm{~km} / \mathrm{hr}$, whereas, for $\mathrm{AC}$ at $100 \%$ penetration, the maximum average speed is about $69.46 \mathrm{~km} / \mathrm{hr}$, almost $15 \%$ higher. Similarly, the maximum flow rate for MC is 2256.51 vehicles/hr, where the maximum flow rate for $\mathrm{AC}$ is 3251.26 vehicles/hr, which is almost $44 \%$ higher. The introduction of AC into the model has resulted in higher and smooth traffic flows, indicating stability and constancy as indicated by the speed versus flow rate graph. Due to their timely maneuverability, the flow is kept constant in comparison to MC speed versus flow rate trend. All of these results indicate a glaring finding that AV can increase road capacity as suggested by previous studies and manufacturers.

3.2. Scenario 2. In this scenario, heterogeneous traffic is introduced by showing different penetration percentage of $\mathrm{AC}$ with MC. The simulation results shown in Figure 3 are expected to show that the combination of $\mathrm{AC}+\mathrm{MC}$ in which $\mathrm{AC}$ composition is $80 \%$ has more stable traffic flows with the highest average speeds under the same density. This proves the concept that if the percentage of AC increases like in the case from $20 \%$ to $80 \%$ on the road networks, road capacity increases until it reaches the critical capacity. For example, in this case, the maximum flow rate for $\mathrm{AC}+\mathrm{MC}$ in which $\mathrm{AC}$ composition is around $20 \%$ is 2433 vehicles/hr, whereas the maximum flow rate for $\mathrm{AC}+\mathrm{MC}$ in which $\mathrm{AC}$ composition is around $80 \%$ is 3013 vehicles $/ \mathrm{hr}$, almost $24 \%$ increase in flow rate. Similarly, the maximum average speed for rate for $\mathrm{AC}+\mathrm{MC}$ in which $\mathrm{AC}$ composition is around $20 \%$ is $62.04 \mathrm{~km} / \mathrm{hr}$, whereas the maximum average speed for $\mathrm{AC}+\mathrm{MC}$ in which $\mathrm{AC}$ composition is around $80 \%$ is $69.51 \mathrm{~km} / \mathrm{hr}$, almost $12 \%$ increase in average speed. This proves that, in the congested phase, the higher percentage of AC composition will be more beneficial than MC. The interesting thing to note here is that, in compositions of AC $40 \%$ and MC $60 \%$ (case 1), the flow rate in free flow phase is less than the composition of AC $20 \%$ and MC $80 \%$ (case 2). This is due to the fact that, in simulation, the values of ALC and PLC are set to 0.5 . This means that, in case 1 , half of AC will follow PLC and the other half will follow ALC. Similarly, half of MC will only be able to change lane by following ALC behavior. Similar rules are applied in case 2 . In this simulation, more MC (40\%) are following ALC behavior and less AC (10\%) are observing PLC behavior compared to the previous simulation in which MC (30\%) are following ALC behavior and AC (20\%) are observing PLC behavior. The unexpected change in results in free flow phase of both simulations lies in the rules of lane 


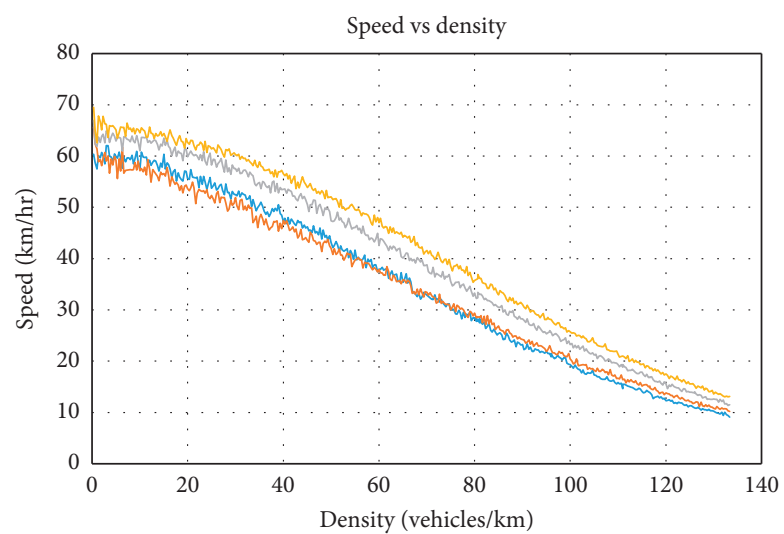

$-\mathrm{AC}+\mathrm{MC}, 20 \% \mathrm{AC} \quad \mathrm{AC}+\mathrm{MC}, 60 \% \mathrm{AC}$

(a)

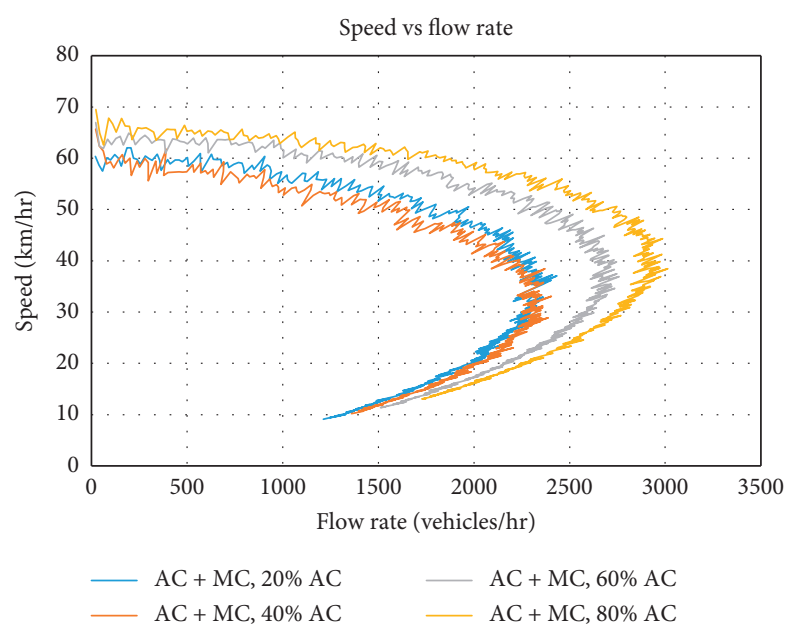

(c)

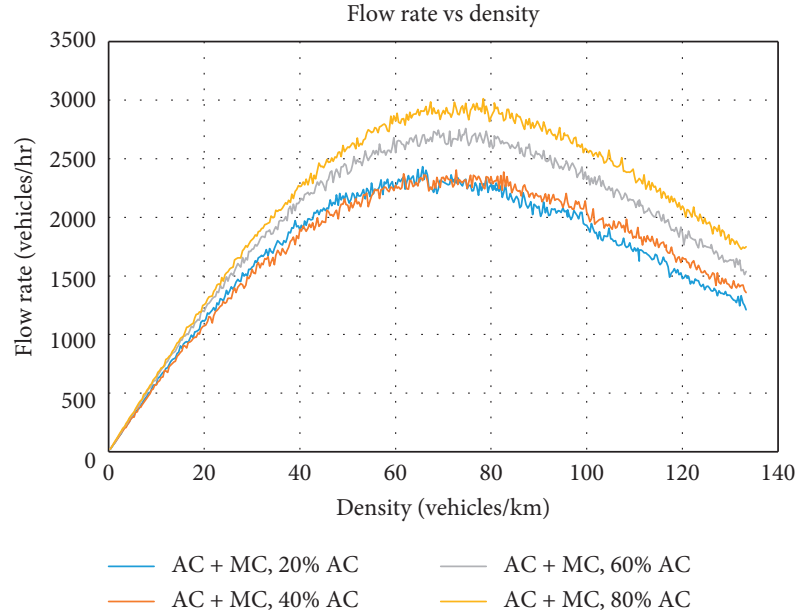

(b) 


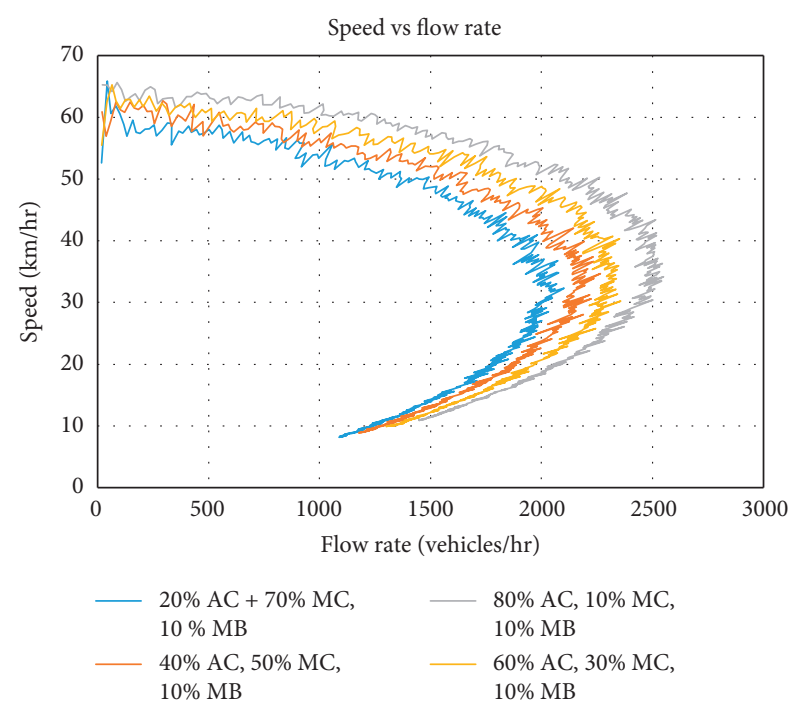

(a)

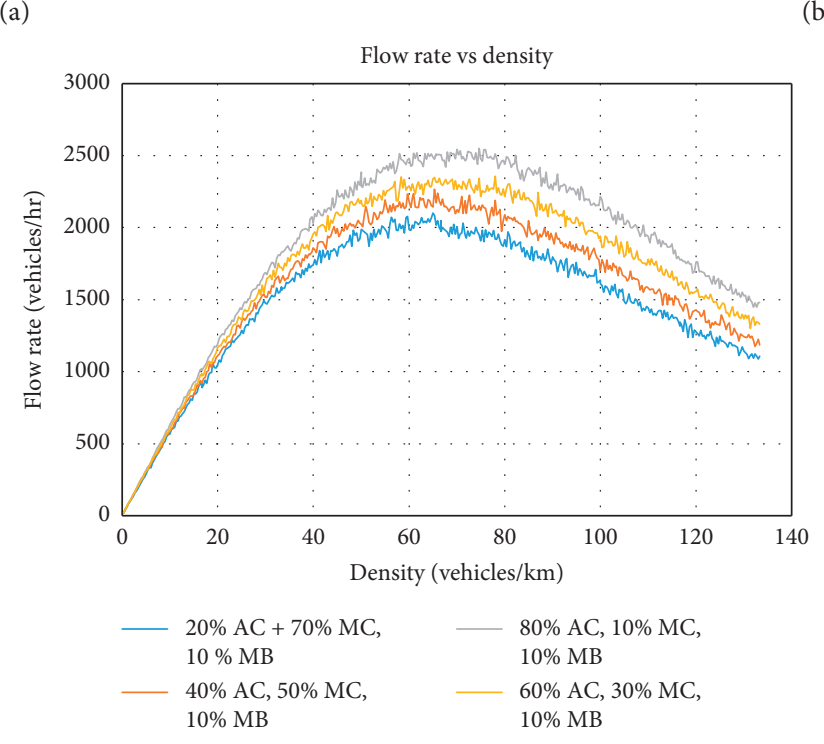

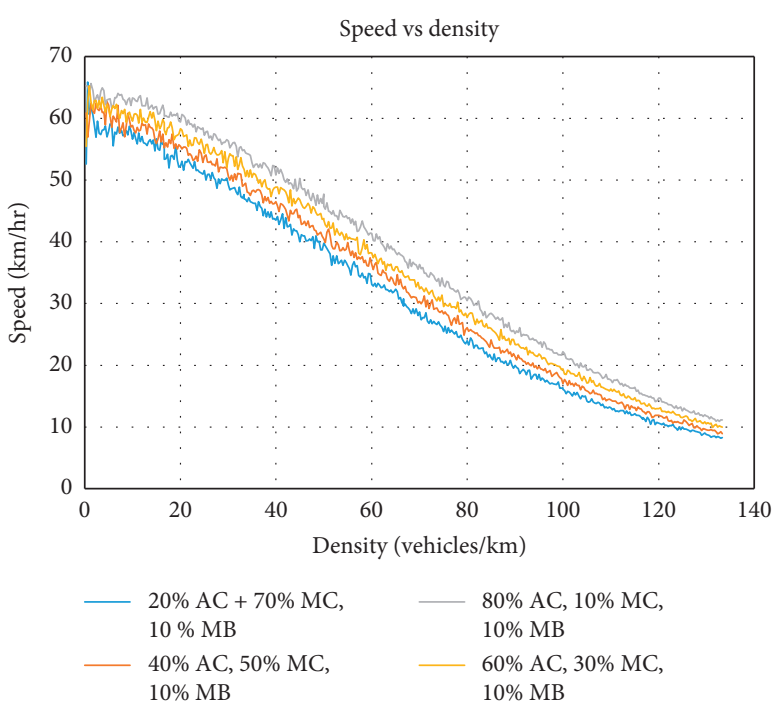

(b)

(c)

Figure 4: Traffic flow fundamental diagrams for different compositions of AC, MC, and MB.

with one significant difference: $\mathrm{MB}$ is replaced with $\mathrm{AB}$ and the percentage of $\mathrm{AB}$ is kept constant at $10 \%$. The percentage of $\mathrm{AB}$ is kept constant at $10 \%$ to indicate the proportion of $\mathrm{AC}$ and $\mathrm{MC}$ in this comparison. It is clear from Figure 5 that when the percentages of $\mathrm{AC}, \mathrm{MC}$, and $\mathrm{AB}$ are at $20 \%, 70 \%$, and $10 \%$, the maximum flow rate and maximum average speeds are 2113 vehicles/hr and $59.15 \mathrm{~km} / \mathrm{hr}$, respectively. On the same hand, when the percentages of $\mathrm{AC}, \mathrm{MC}$, and $\mathrm{AB}$ are at $80 \%, 10 \%$, and $10 \%$, the maximum flow rate and maximum average speeds are 2882 vehicles $/ \mathrm{hr}$ and $71.08 \mathrm{~km} / \mathrm{hr}$, respectively. There is an increase in the flow rate of about $36 \%$ and there is an increase in the maximum average speed of about $20 \%$ when the $\mathrm{AC}$ is at $80 \%$. Furthermore, this also presents an interesting observation similar to the results of scenario 2 . In simulation of $\mathrm{AC} 40 \%, \mathrm{MC} 50 \%$, and $\mathrm{AB} 10 \%$ (case 1 ), the flow rate in free flow phase is close to $\mathrm{AC} 80 \%, \mathrm{MC}$
$10 \%$, and $\mathrm{AB} 10 \%$ (case 2 ). The reasons as mentioned in scenario 2 again lie in the rules of ALC and PLC. In case 1, more vehicles of MC will follow ALC behavior, which means that both $\mathrm{AC}$ and $\mathrm{MC}$ will be able to change lanes efficiently in free flow phase. However, in congestion phase, less penetration rate of AC will not be able to abate the traffic congestion as shown in case 2 . This point is again highlighted in the simulation results of AC 60\%, MC 30\%, and $\mathrm{AB} 10 \%$ (case 3 ). Since there are less $\mathrm{MC}$ in case 3 in free flow phase, less MC will follow ALC behavior in comparison to case 1 . As a result, the flow rate in free flow phase of case 3 will be less than case 1 . In the congestion phase, the high penetration rate of $\mathrm{AC}$ in case 2 and case 3 will allow them to change lanes more efficiently than case 1 due to the fact that the flow rate in case 2 and case 3 is higher than case 1 . This further reinforces the finding of scenario 2 that ALC is also a deciding factor in increasing 


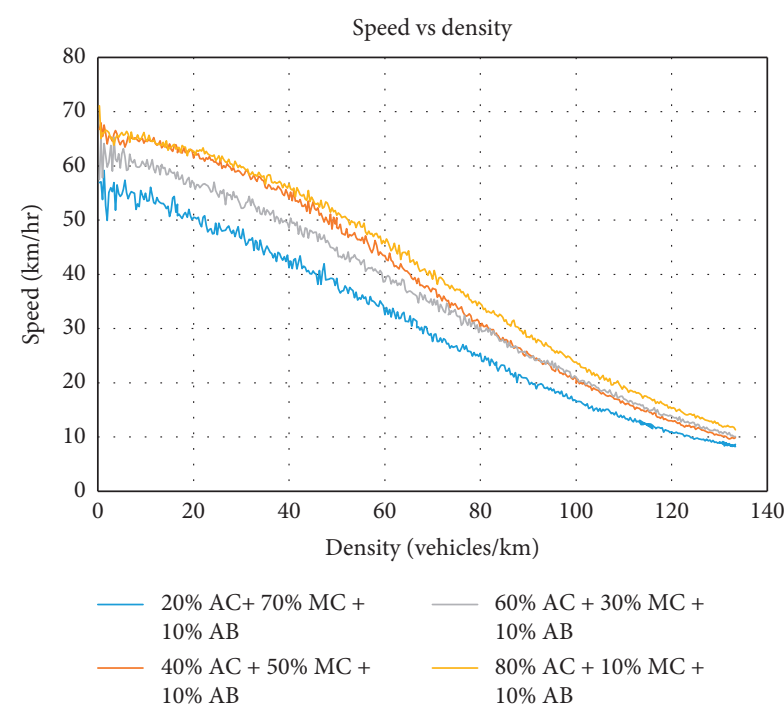

(a)

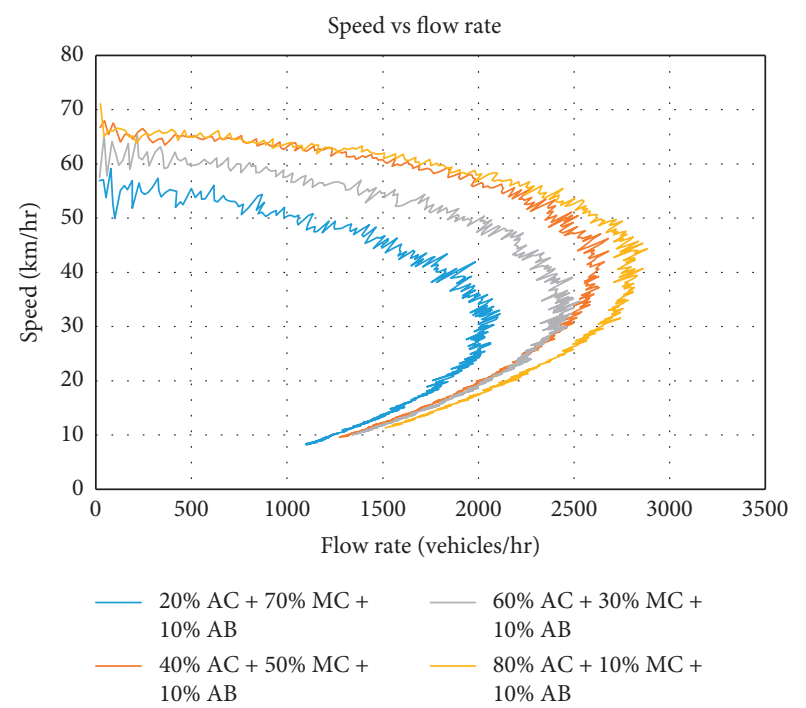

(b)

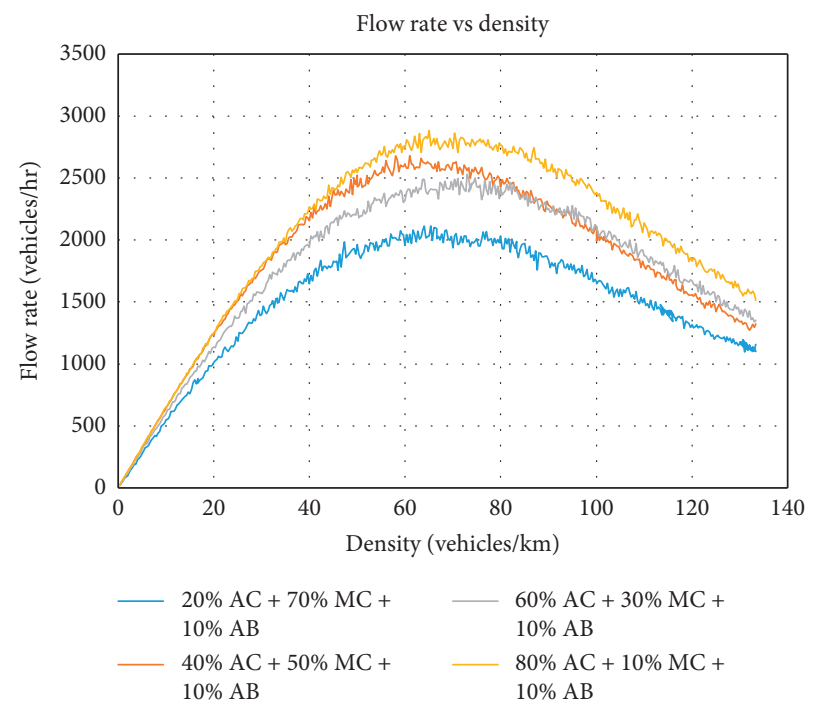

(c)

Figure 5: Traffic flow fundamental diagrams for different compositions of $\mathrm{AC}, \mathrm{MC}$, and $\mathrm{AB}$.

the flow rate. Moreover, the high penetration rate of AC increases the flow rate in congestion phase as they will be able to change lanes more efficiently due to their less reaction time and less headway. Merely including the AC (until a certain threshold) will not significantly increase the flow rate in free flow phase. As stated before, the importance of ALC is tested through scenarios 6 to 9 .

3.5. Scenario 5. In scenario $5, \mathrm{AB}$ and $\mathrm{MB}$ are introduced as a part of heterogeneous traffic. Different compositions are simulated to infer the most feasible situation. As shown in Figure 6, the results clearly delineate that the compositions of $\mathrm{AC}$ and $\mathrm{AB}(90 \% \mathrm{AC}$ and $10 \% \mathrm{AB})$ outdo other arrangements. Thus, if $\mathrm{AB}$ on road networks are introduced instead of $\mathrm{MB}$, the flow rate will increase significantly as shown by the peak flow rate in Figure 3. The maximum flow rate for $\mathrm{MC}+\mathrm{MB}$ in which $\mathrm{MB}$ composition is around
$10 \%$ is 1735 vehicles/hr. The maximum flow rate for $\mathrm{AC}+\mathrm{AB}$ in which $\mathrm{AB}$ composition is around $10 \%$ is 2947 vehicles $/ \mathrm{hr}$, almost $70 \%$ noteworthy increase in flow rate, thus indicating the increase in road capacity if $A B$ is introduced with $\mathrm{AC}$. It is expected that more passengers will be inclined to use $A B$ due to its safety and flexibility features. Similarly, the maximum average speed for for $\mathrm{MC}+\mathrm{MB}$ in which $\mathrm{MB}$ composition is around $10 \%$ is $56.36 \mathrm{~km} / \mathrm{hr}$, whereas the maximum average speed for $\mathrm{AC}+\mathrm{AB}$ in which $\mathrm{AB}$ composition is around $10 \%$ is $67.94 \mathrm{~km} / \mathrm{hr}$, almost $20 \%$ increase in average speed as the reaction delays of $A B$ will be the same as those of $A C$. If we compare the $\mathrm{MC}+\mathrm{AB}$ model with $\mathrm{MC}+\mathrm{MB}$ model, it is evidently clear that, with only $10 \%$ introduction of $\mathrm{AB}$ into the model, the flow rate increases. The free flow phase is similar in the start but as the density increases, the flow rate in $\mathrm{MC}+\mathrm{AB}$ composition is more stable even in the congested phase. 


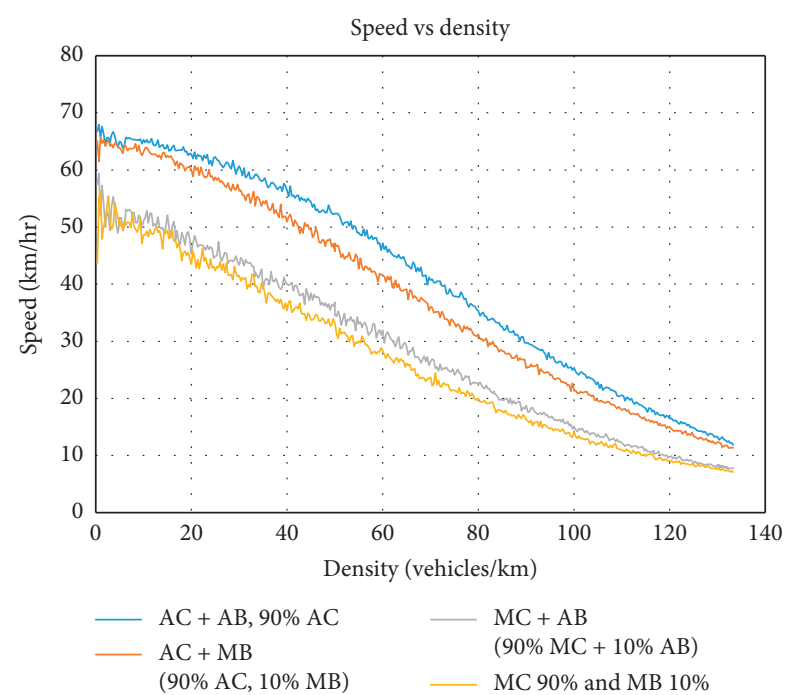

(a)

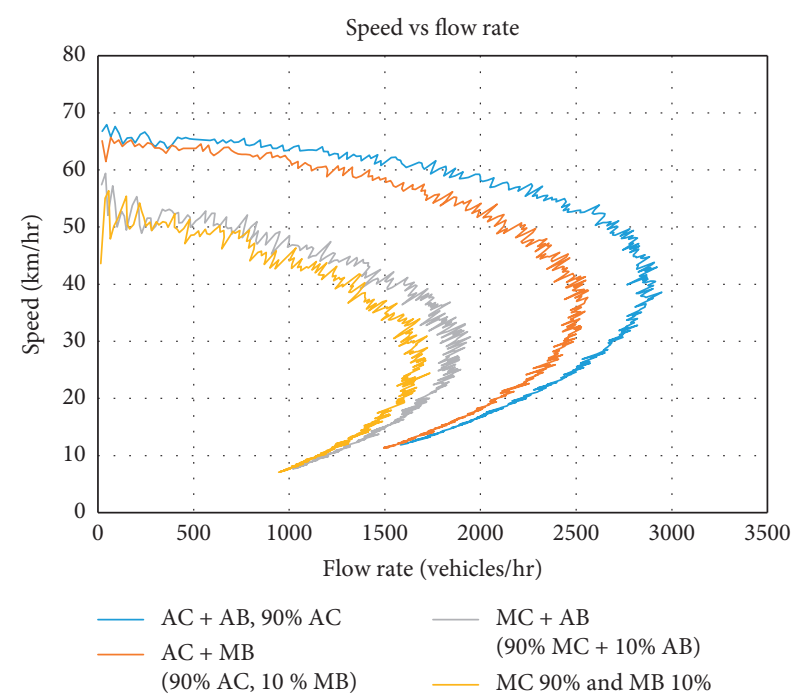

(b)

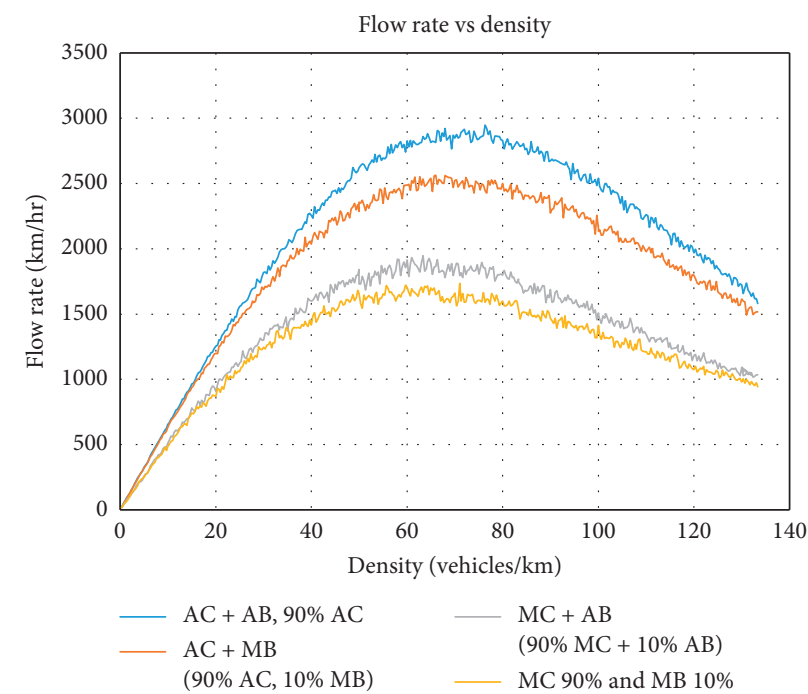

(c)

Figure 6: Traffic flow fundamental diagrams for different compositions of AC, MC, AB, and MB.

Thus, with an only penetration rate of $10 \%, \mathrm{AB}$ can have a significant positive impact on overall road capacity.

The main findings of scenarios 1 to 5 can be summarized as follows:

(a) With the increase of penetration percentage of $\mathrm{AC}$ and $A B$ in cars, the overall flow rate of the network increases, thus indicating a significant increase in capacity.

(b) As shown in the above figures, for the same values of density, the involvement of AC and AB in the model increases the free flow phase of the network, which leads to stable traffic flows for a larger duration of time.

(c) The use of $\mathrm{AB}$ in the traffic streams alongside $\mathrm{AC}$ will be accommodating in abating the traffic congestions as it is generally expected that the $\mathrm{AC}$ will be used in carpooling. If $\mathrm{AB}$ is employed concurrently with $\mathrm{AC}$, not only will the capacity of network increase but also more passengers can be accommodated and can be incentivized to use the features of $A B$. Thus, $A B$ has the potential to reduce traffic congestion considerably if implemented duly.

3.6. Scenario 6. In this scenario, 4 different types of vehicles, that is, $\mathrm{AC}, \mathrm{MC}, \mathrm{AB}$, and $\mathrm{MB}$, are introduced in the model at $45 \%, 45 \%, 5 \%$, and $5 \%$, respectively. This arrangement is simulated in the model by changing the values of ALC and PLC. The PLC in this scenario is kept constant at 0 , whereas ALC is changed four times in each composition, that is, 0.2 , $0.4,0.6$, and 0.8. As shown in Figure 7, the trend when values of ALC fall in the range from 0.4 to 0.8 is generally the same for all graphs with the exception when ALC is 0.2 . This is due to the fact that only $20 \%$ of all the vehicles are changing their lanes aggressively, whereas the rest are not 


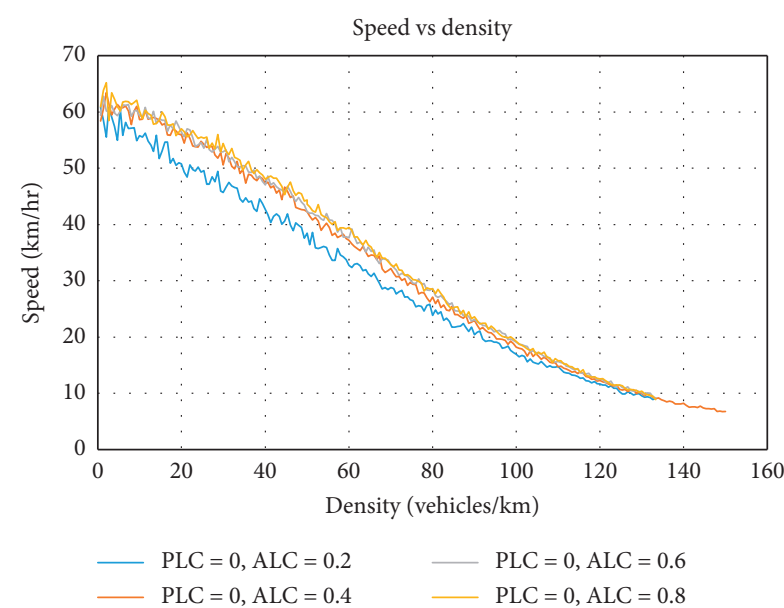

(a)

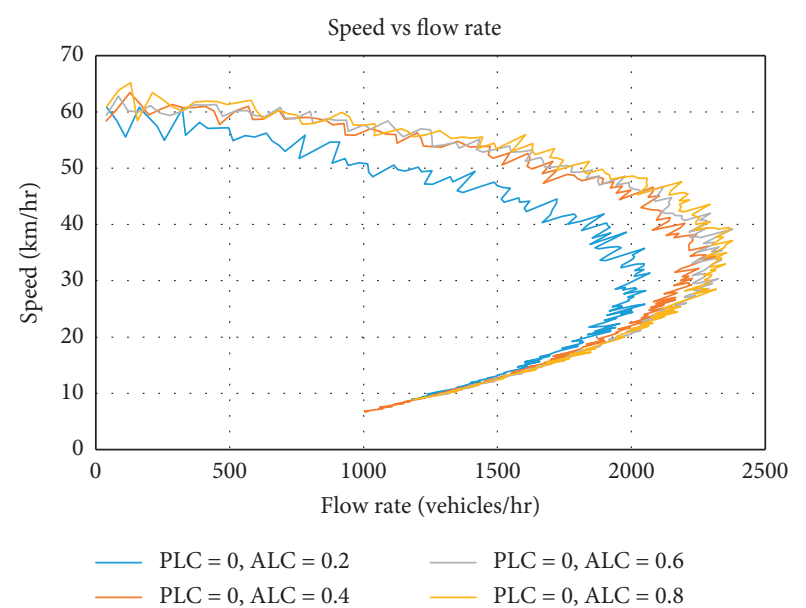

(b)

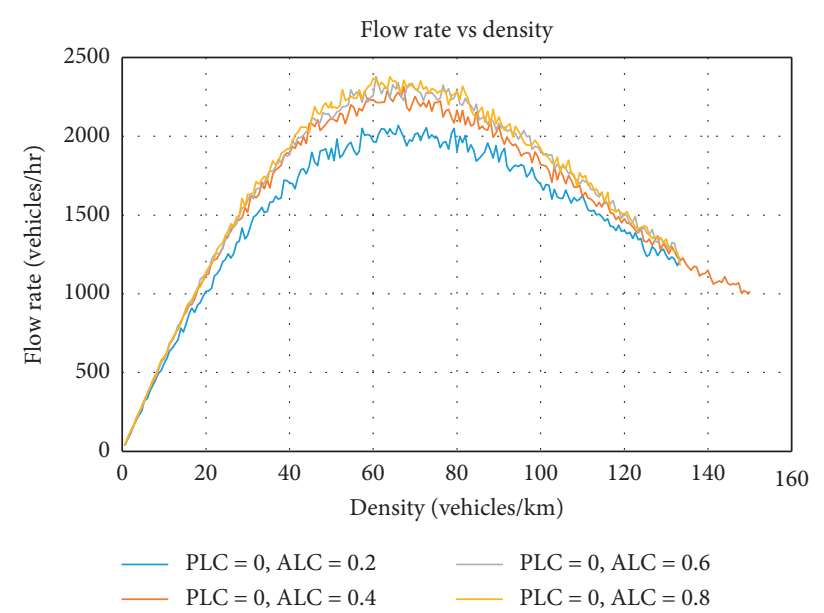

(c)

Figure 7: Traffic flow fundamental diagrams for different compositions of AC, MC, AB, and MB with different values of ALC when PLC is fixed at 0 .

modeled to change their lane. Therefore, the flow rate is less compared to other values. If the vehicles do not decide to change their lanes, then, under the same density, it is expected that the flow rate will fall considerably as the number of vehicles will increase substantially. The key point to notice here is that even when the value of ALC is 0.8 , that is, $80 \%$, vehicles, both manual and autonomous, are changing their lanes aggressively; the overall trend with other simulations when ALC is 0.4 and 0.6 is more or less the same. Thus, increasing the aggressiveness of drivers does not increase the flow rate after a certain threshold is reached.

3.7. Scenario 7. As mentioned before, this scenario is similar to scenario 6 in terms of vehicular layout with 4 different types of vehicles. In this scenario, the value of ALC is kept constant at 0 while changing the values of PLC four different times in each simulation from 0.2 to 0.8 . The trend of all the simulations is the same in free flow and congested phase as illustrated in Figure 8. Since ALC is 0 here, no manual vehicle will change its lane. As a result, the flow rate is less compared to other simulations. Thus, increasing the polite behavior of autonomous vehicles will not dramatically increase the flow rate after a critical value is reached. The free flow phase and congested flow phase are the same in this range.

3.8. Scenario 8. In this scenario, again the vehicular arrangement is kept as same as that of scenarios 6 and 7 . However, no value of ALC and PLC is kept constant. Instead, in every simulation, different values of PLC and ALC are used in the range from 0.2 to 0.8 . For example, when ALC is 0.2 , PLC is 0.8 and vice versa. The results of all simulations show similar trends as shown in Figure 9 with no drastic changes between them. At the start of simulation when the value of PLC is 0.4 and ALC is 0.6 , the average speed of vehicles is slightly less than other simulations but it reaches the flow rate and speed of other simulations when density crosses the threshold of 40 vehicles/ $\mathrm{km}$. After this threshold, the speed and flow rate of all the values exhibit similar patterns and trends. Thus, it can be said in the congested phase that all simulations produce more or less 


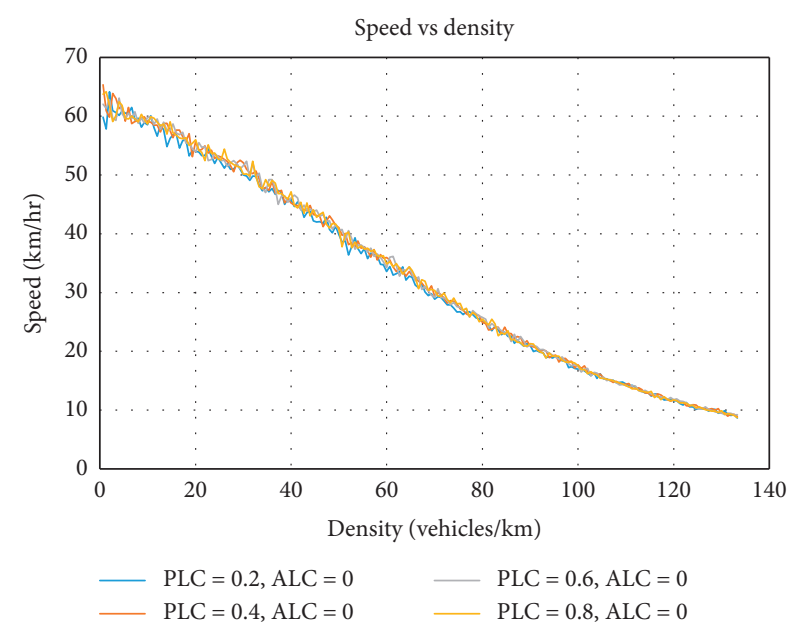

(a)

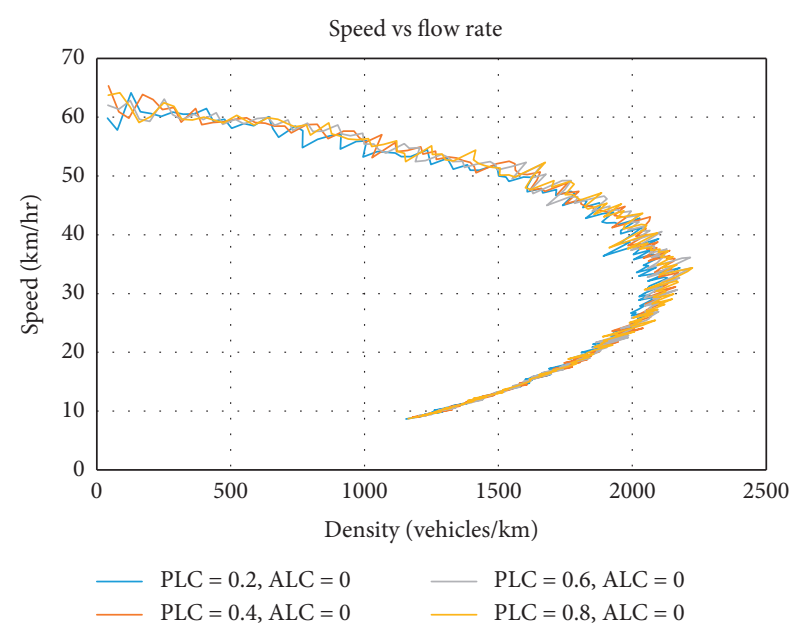

(b)

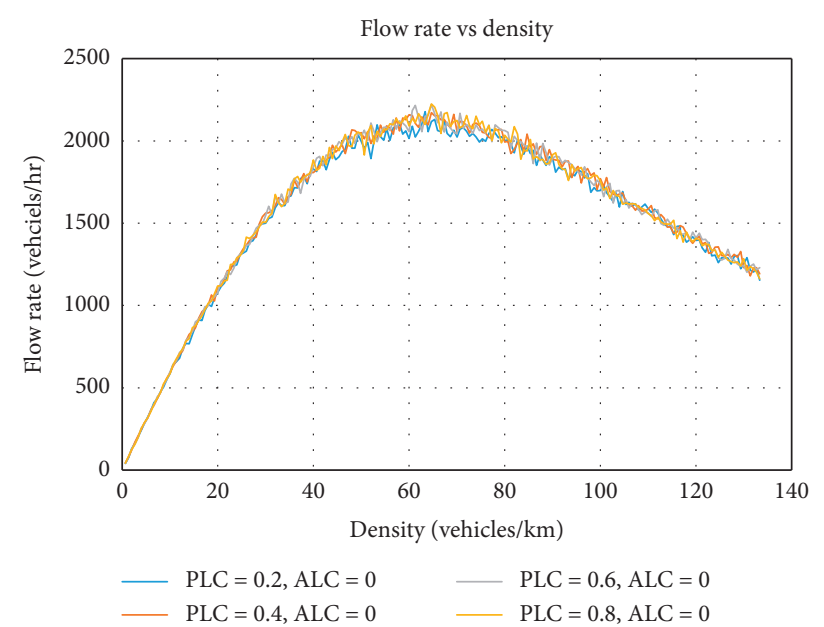

(c)

Figure 8: Traffic flow fundamental diagrams for different compositions of AC, MC, AB, and MB with different values of PLC when ALC is fixed at 0 .

similar outputs and changing the values of ALC and PLC does not reveal radical variations.

3.9. Scenario 9. This is the last comparative scenario in this study. In this scenario, the vehicular compositions are the same as those of previous scenarios from 6 to 8 . Three different types of simulations are run with three unique values of ALC and PLC in each simulation and the results are shown in Figure 10. In the first simulation, ALC is 0 and PLC is 1 . In the second simulation, PLC is 1 and ALC is 0 , and, in the third simulation, both values are 0 . The results of all of the simulations are somewhat expected. Traffic is more stable with high flow rates in free flow and congested flow phase when ALC is 1 and PLC is 0 . This is due to the fact that both autonomous and manual vehicles will follow an aggressive lane changing maneuver, which will subsequently increase the capacity of the road. When PLC is 1 and ALC is 0 , only autonomous vehicles will be allowed to change their lanes by following the polite lane changing maneuver as manual vehicles in this study will only follow the aggressive lane changing behavior. As a result, the flow rate is less because almost half of the vehicles (manual) will not be able to change their lanes and will travel on the same lane irrespective of the gaps provided. When ALC and PLC are both 0 , the results are on par with the simulation when PLC was 1 and ALC was 0 . No vehicle will change lane and will remain on the same path regardless of the acceptable gaps. Thus, the road capacity of any link can be increased if the vehicles are allowed to follow the aggressive routine.

The main findings of scenarios 6 to 9 can be summarized as follows:

(a) If the vehicular compositions are kept constant and only values of ALC and PLC are changed, there are no sweeping fluctuations in the overall road capacity.

(b) Only when the vehicles are allowed to change the lanes aggressively will there be a vast rise in flow rate and capacity of the network. Polite lane change does not significantly affect the flow rate and its impact is 


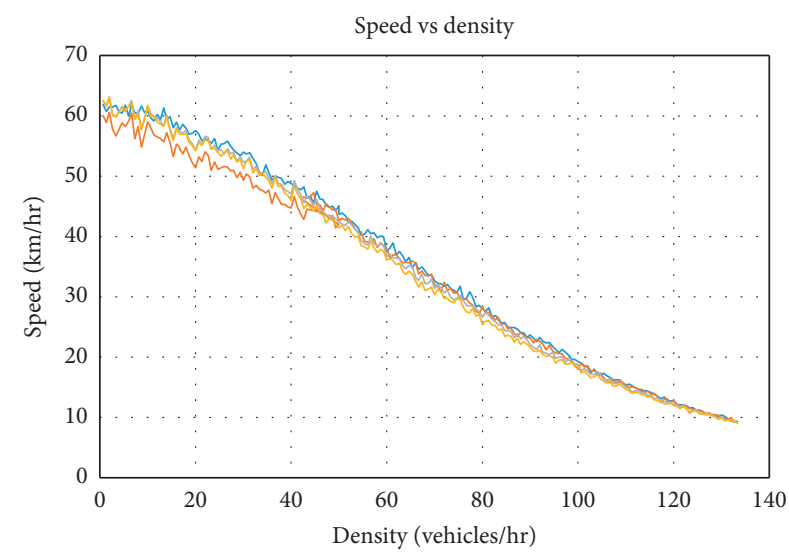

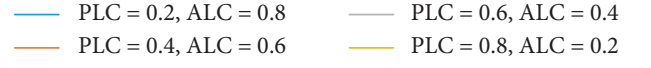

(a)

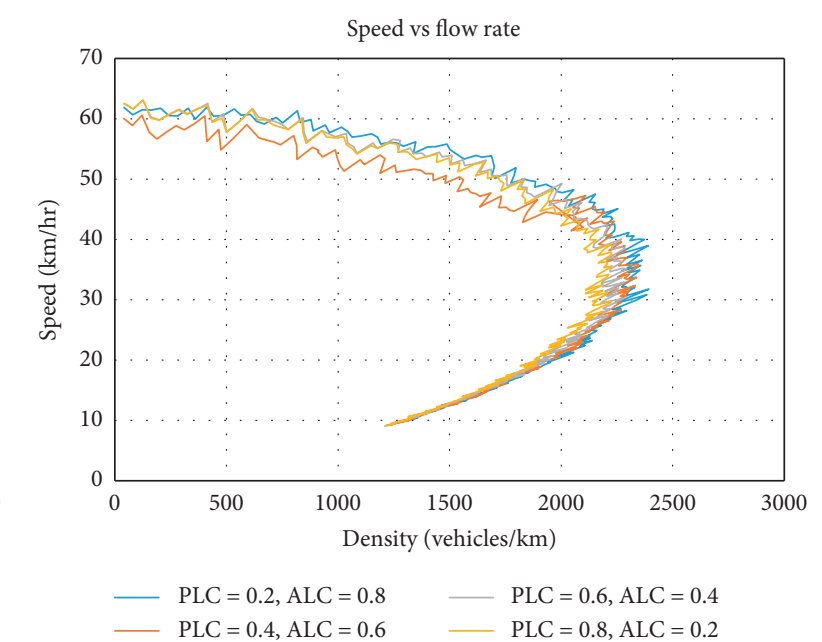

(b)

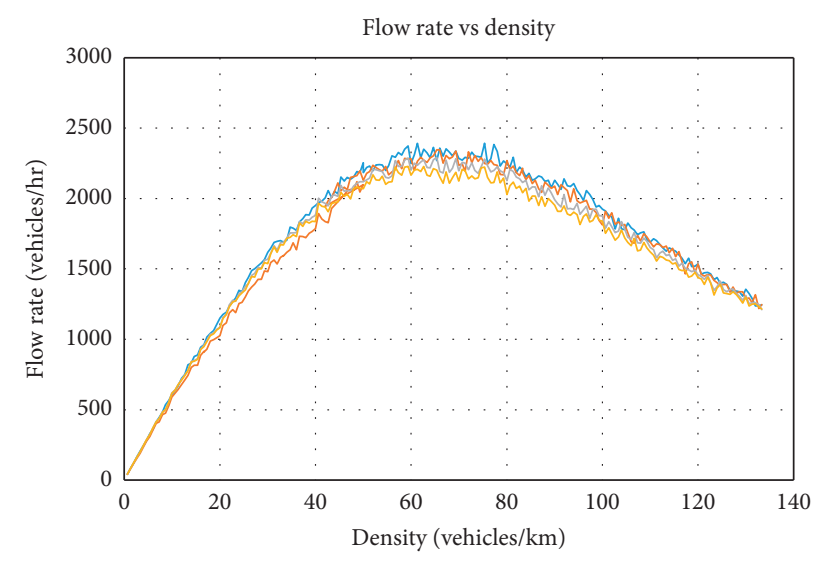

$\longrightarrow \mathrm{PLC}=0.2, \mathrm{ALC}=0.8 \quad \mathrm{PLC}=0.6, \mathrm{ALC}=0.4$
$\mathrm{PLC}=0.4, \mathrm{ALC}=0.6 \quad \mathrm{PLC}=0.8, \mathrm{ALC}=0.2$

(c)

Figure 9: Traffic flow fundamental diagrams for different compositions of AC, MC, AB, and MB with different values of PLC and ALC.

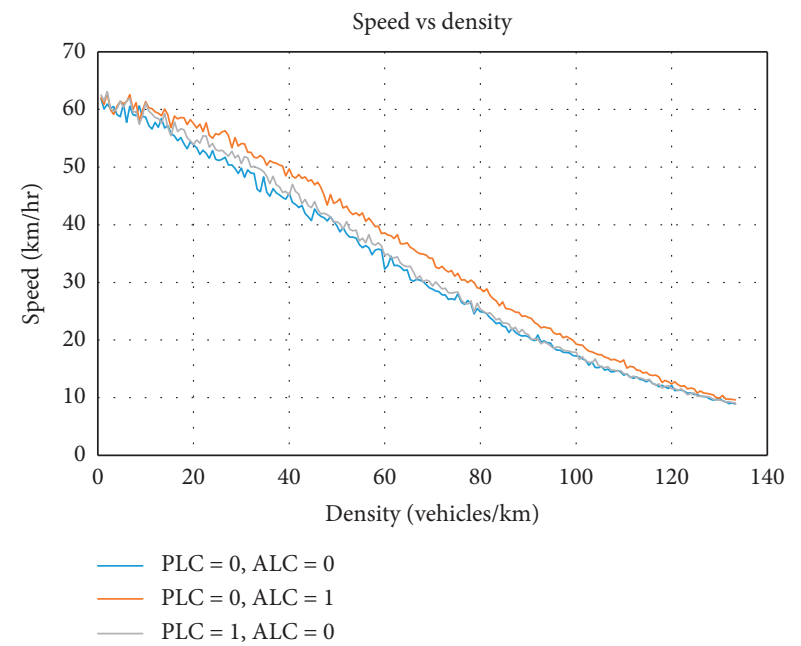

(a)

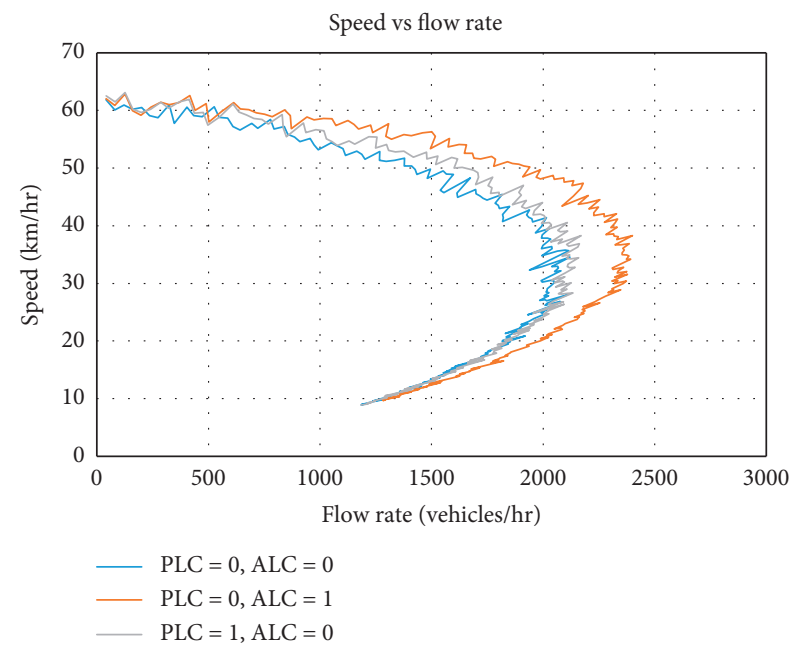

(b)

Figure 10: Continued. 


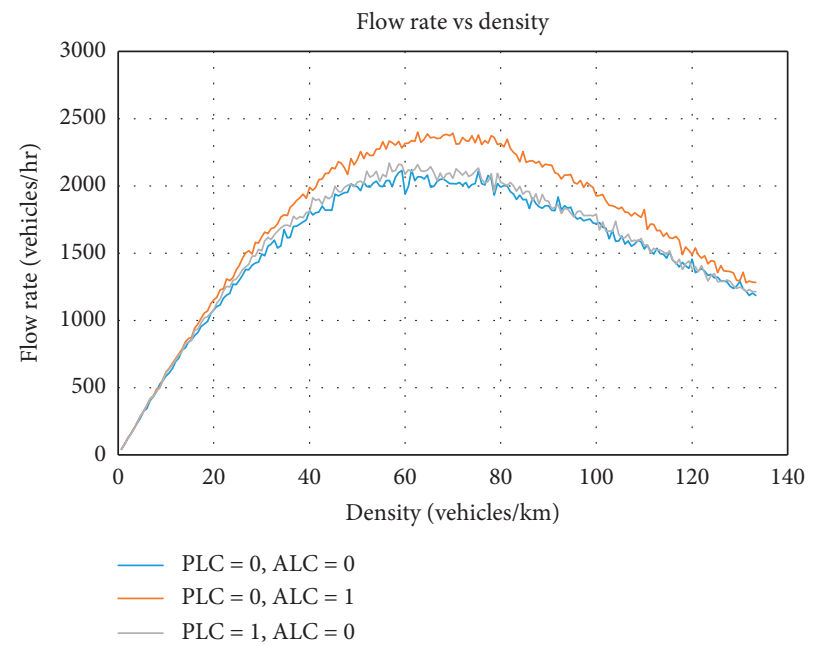

(c)

Figure 10: Traffic flow fundamental diagrams for different compositions of AC, MC, AB, and MB with different values of PLC and ALC.

TABLE 7: Details of different scenarios.

\begin{tabular}{lc}
\hline Scenarios & Details/composition in model \\
\hline Scenario 1 & AC $100 \%$ and MC $100 \%$ (PLC and ALC $=0.5)$ \\
Scenario 2 & Different compositions of AC and MC (PLC and ALC =0.5) \\
Scenario 3 & Different compositions of AC, MC, and MB (PLC and ALC=0.5) \\
Scenario 4 & Different compositions of AC, MC, and AB (PLC and ALC =0.5) \\
Scenario 5 & Different compositions of AC, MC, AB, and MB (PLC and ALC =0.5) \\
Scenario 6 & Different compositions of AC, MC, AB, and MB with different values of ALC when PLC is fixed at 0 \\
Scenario 7 & Different compositions of AC, MC, AB, and MB with different values of PLC when ALC is fixed at 0 \\
Scenario 8 & Different compositions of AC, MC, AB, and MB with different values of PLC and ALC \\
Scenario 9 & Different compositions of AC, MC, AB, and MB with different values of PLC and ALC \\
\hline
\end{tabular}

more or less equivalent to the setting when none of the vehicles are allowed to change as polite lane change requires certain and specific requirements to be met before it is implemented.

\section{Conclusion}

In this paper, we have studied the heterogeneity in traffic by comparing different penetration rates of four different types of vehicles, that is, autonomous cars, autonomous buses, manual cars, and manual buses. For this purpose, a revised CA model was developed as explained in previous model description phase of this paper. The characteristics and parameters of all four vehicles were defined. Furthermore, two sets of lane changing behavior (aggressive lane changing and polite lane changing) were also incorporated in the model with the assumption that manual vehicles will only follow ALC and autonomous vehicles will follow both PLC and ALC. In order to deeply understand the notion of mixed traffic flows, different scenarios through different compositions of vehicles were devised and simulated.

The results indicated that, with an increase in the penetration rate of autonomous vehicles, the overall flow rate and average speed increase. The introduction of the autonomous bus is a critical factor in increasing the effectiveness of road capacity as not only will the flow rate of traffic increase but also more passengers can be accommodated. It is expected that, with the introduction of $\mathrm{AC}$ into the market, $\mathrm{AB}$ will also follow up and will be immensely obliging in the overall stability of traffic flows. Moreover, it was also noted that if the vehicular configurations are assumed to be continual and only values of ALC and PLC are changed, there are no extensive variabilities on the overall road capacity. The traffic flow rate surges considerably only when all the vehicles follow ALC. Conversely, if only PLC is allowed, the difference in flow rate is trifling.

Future work on modeling of signalized and unsignalized junctions under different compositions of heterogeneous traffic needs to be addressed.

\section{Data Availability}

Since there are no any real data related to autonomous, we use only simulation data in this paper. The simulation data are available from the corresponding author upon request.

\section{Conflicts of Interest}

The authors declare that they have no conflicts of interest. 


\section{Acknowledgments}

This research was funded by the "Research of Transportation Strategy for Powerful Nation" (2017ZD07) and the major consulting project of the Chinese Academy of Engineering (no. 2017ZD07); special gratitude shall be extended to Dr. Ruimin Li, Miss Shumaila Qadeer, Miss Faiza Naveed, Miss Sadia, and Palwisha for their invaluable suggestions.

\section{References}

[1] S. Pawar, S. Gupte, and K. Bhatt, "Traffic congestion-cause and solutions: a case study of Hadapsar road, Magarpatta, Pune," International Journal of Scientific Research in Science, Engineering and Technology, vol. 4, 2018.

[2] World Health Organization, "Global status report on road safety 2018," World Health Organization, Geneva, Switzerland, 2018, https://www.who.int/violence_injury_prevention/ road_safety_status/2018/en/.

[3] A. Talebpour and H. S. Mahmassani, "Influence of connected and autonomous vehicles on traffic flow stability and throughput," Transportation Research Part C: Emerging Technologies, vol. 71, pp. 143-163, 2016.

[4] B. Friedrich, "The effect of autonomous vehicles on traffic,"Autonomous Driving, Springer, pp. 317-334, Berlin, Germany, 2016.

[5] D. J. Fagnant and K. Kockelman, "Preparing a nation for autonomous vehicles: opportunities, barriers and policy recommendations," Transportation Research Part A: Policy and Practice, vol. 77, pp. 167-181, 2015.

[6] J. Ma, X. Li, F. Zhou, J. Hu, and B. B. Park, "Parsimonious shooting heuristic for trajectory design of connected automated traffic part II: computational issues and optimization," Transportation Research Part B: Methodological, vol. 95, pp. 421-441, 2017.

[7] F. Zhou, X. Li, and J. Ma, "Parsimonious shooting heuristic for trajectory design of connected automated traffic part I: theoretical analysis with generalized time geography," Transportation Research Part B: Methodological, vol. 95, pp. 394-420, 2017.

[8] M. W. Levin and S. D. Boyles, "A multiclass cell transmission model for shared human and autonomous vehicle roads," Transportation Research Part C: Emerging Technologies, vol. 62, pp. 103-116, 2016.

[9] U. Khan, P. Basaras, L. Schmidt-Thieme, A. Nanopoulos, and D. Katsaros, "Analyzing cooperative lane change models for connected vehicles," in Proceedings of the 2014 International Conference on Connected Vehicles and Expo (ICCVE), pp. 565-570, Vienna, Austria, November 2014.

[10] L. Du, L. Han, and S. Chen, "Coordinated online in-vehicle routing balancing user optimality and system optimality through information perturbation," Transportation Research Part B: Methodological, vol. 79, pp. 121-133, 2015.

[11] X. Qu, S. Wang, and J. Zhang, "On the fundamental diagram for freeway traffic: a novel calibration approach for singleregime models," Transportation Research Part B: Methodological, vol. 73, pp. 91-102, 2015.

[12] P. Tientrakool, Y.-C. Ho, and N. F. Maxemchuk, "Highway capacity benefits from using vehicle-to-vehicle communication and sensors for collision avoidance," in Proceedings of 2011 IEEE Vehicular Technology Conference (VTC Fall), pp. 1-5, San Francisco, CA, USA, September 2011.
[13] B. Van Arem, C. J. G. Van Driel, and R. Visser, "The impact of cooperative adaptive cruise control on traffic-flow characteristics," IEEE Transactions on Intelligent Transportation Systems, vol. 7, no. 4, pp. 429-436, 2006.

[14] P. Hidas, "Modelling vehicle interactions in microscopic simulation of merging and weaving," Transportation Research Part C: Emerging Technologies, vol. 13, no. 1, pp. 37-62, 2005.

[15] Z. Zheng, S. Ahn, D. Chen, and J. Laval, "Freeway traffic oscillations: microscopic analysis of formations and propagations using wavelet transform," Procedia-Social and Behavioral Sciences, vol. 17, pp. 702-716, 2011.

[16] J. C. McCall, D. P. Wipf, M. M. Trivedi, and B. D. Rao, "Lane change intent analysis using robust operators and sparse bayesian learning," IEEE Transactions on Intelligent Transportation Systems, vol. 8, no. 3, pp. 431-440, 2007.

[17] P. G. Gipps, "A model for the structure of lane-changing decisions," Transportation Research Part B: Methodological, vol. 20, no. 5, pp. 403-414, 1986.

[18] K. Ahmed, M. Ben-Akiva, H. Koutsopoulos, and R. Mishalani, "Models of freeway lane changing and gap acceptance behavior," Transportation and Traffic Theory, vol. 13, pp. 501515, 1996.

[19] A. Kesting, M. Treiber, and D. Helbing, "General lanechanging model MOBIL for car-following models," Transportation Research Record: Journal of the Transportation Research Board, vol. 1999, no. 1, pp. 86-94, 2007.

[20] Y. Du, Y. Wang, and C.-Y. Chan, "Autonomous lane-change controller via mixed logical dynamical," in Proceedings of the 17th International IEEE Conference on Intelligent Transportation Systems (ITSC), pp. 1154-1159, Qingdao, China, October 2014.

[21] A. Tawari, S. Sivaraman, M. M. Trivedi, T. Shannon, and M. Tippelhofer, "Looking-in and looking-out vision for urban intelligent assistance: estimation of driver attentive state and dynamic surround for safe merging and braking," in Proceedings of the 2014 IEEE Intelligent Vehicles Symposium Proceedings, pp. 115-120, Dearborn, MI, USA, June 2014.

[22] Y. Hou, P. Edara, and C. Sun, "Situation assessment and decision making for lane change assistance using ensemble learning methods," Expert Systems with Applications, vol. 42, no. 8, pp. 3875-3882, 2015.

[23] J. Hu, L. Kong, W. Shu, and M.-Y. Wu, "Scheduling of connected autonomous vehicles on highway lanes," in Proceedings of the 2012 IEEE Global Communications Conference (GLOBECOM), pp. 5556-5561, Anaheim, CA, USA, December 2012.

[24] T. Awal, M. Murshed, and M. Ali, "An efficient cooperative lane-changing algorithm for sensor- and communicationenabled automated vehicles," in Proceedings of the 2015 IEEE Intelligent Vehicles Symposium (IV), pp. 1328-1333, Seoul, South Korea, June 2015.

[25] T. Awal, L. Kulik, and K. Ramamohanrao, "Optimal traffic merging strategy for communication- and sensor-enabled vehicles," in Proceedings of the 16th International IEEE Conference on Intelligent Transportation Systems (ITSC 2013), pp. 1468-1474, The Hague, Netherlands, October 2013.

[26] D. Desiraju, T. Chantem, and K. Heaslip, "Minimizing the disruption of traffic flow of automated vehicles during lane changes," IEEE Transactions on Intelligent Transportation Systems, vol. 16, no. 3, pp. 1249-1258, 2015.

[27] K. Nagel and M. Schreckenberg, "A cellular automaton model for freeway traffic," Journal de physique I, vol. 2, no. 12, pp. 2221-2229, 1992. 
[28] M. Fukui and Y. Ishibashi, "Traffic flow in 1D cellular automaton model including cars moving with high speed," Journal of the Physical Society of Japan, vol. 65, no. 6, pp. 1868-1870, 1996.

[29] D. Chowdhury, L. Santen, and A. Schadschneider, "Statistical physics of vehicular traffic and some related systems," Physics Reports, vol. 329, no. 4-6, pp. 199-329, 2000.

[30] A. Schadschneider and M. Schreckenberg, "Traffic flow models with "slow-to-start" rules," Annalen der Physik, vol. 509, no. 7, pp. 541-551, 1997.

[31] R. Barlovic, L. Santen, A. Schadschneider, and M. Schreckenberg, "Metastable states in cellular automata for traffic flow," The European Physical Journal B, vol. 5, no. 3, pp. 793-800, 1998.

[32] W. Knospe, L. Santen, A. Schadschneider, and M. Schreckenberg, "Towards a realistic microscopic description of highway traffic," Journal of Physics A: Mathematical and General, vol. 33, no. 48, pp. L477-L485, 2000.

[33] X. Li, Q. Wu, and R. Jiang, "Cellular automaton model considering the velocity effect of a car on the successive car," Physical Review E, vol. 64, Article ID 066128, 2001.

[34] M. Rickert, K. Nagel, M. Schreckenberg, and A. Latour, "Two lane traffic simulations using cellular automata," Physica A: Statistical Mechanics and Its Applications, vol. 231, no. 4, pp. 534-550, 1996.

[35] P. Wagner, K. Nagel, and D. E. Wolf, "Realistic multi-lane traffic rules for cellular automata," Physica A: Statistical Mechanics and Its Applications, vol. 234, no. 3-4, pp. 687-698, 1997.

[36] D. Chowdhury, D. E. Wolf, and M. Schreckenberg, "Particle hopping models for two-lane traffic with two kinds of vehicles: effects of lane-changing rules," Physica A: Statistical Mechanics and Its Applications, vol. 235, no. 3-4, pp. 417-439, 1997.

[37] K. Nagel, D. E. Wolf, P. Wagner, and P. Simon, "Two-lane traffic rules for cellular automata: a systematic approach," Physical Review E, vol. 58, no. 2, pp. 1425-1437, 1998.

[38] W. Knospe, L. Santen, A. Schadschneider, and M. Schreckenberg, "Disorder effects in cellular automata for two-lane traffic," Physica A: Statistical Mechanics and Its Applications, vol. 265, no. 3-4, pp. 614-633, 1999.

[39] X.-G. Li, B. Jia, Z.-Y. Gao, and R. Jiang, "A realistic two-lane cellular automata traffic model considering aggressive lanechanging behavior of fast vehicle," Physica A: Statistical Mechanics and Its Applications, vol. 367, pp. 479-486, 2006.

[40] K. Gao, R. Jiang, S.-X. Hu, B.-H. Wang, and Q.-S. Wu, "Cellular-automaton model with velocity adaptation in the framework of Kerner's three-phase traffic theory," Physical Review E, vol. 76, Article ID 026105, 2007.

[41] C. Tao, J. Bin, L. Xin-Gang, J. Rui, and G. Zi-You, "Synchronized flow in a cellular automaton model with time headway dependent randomization," Chinese Physics Letters, vol. 25, no. 8, pp. 2795-2798, 2008.

[42] O. K. Tonguz, W. Viriyasitavat, and F. Bai, "Modeling urban traffic: a cellular automata approach," IEEE Communications Magazine, vol. 47, 2009.

[43] S. Das, "Cellular automata based traffic model that allows the cars to move with a small velocity during congestion," Chaos, Solitons \& Fractals, vol. 44, no. 4-5, pp. 185-190, 2011.

[44] N. Moussa and A. K. Daoudia, "Numerical study of two classes of cellular automaton models for traffic flow on a twolane roadway," The European Physical Journal B-Condensed Matter, vol. 31, no. 3, pp. 413-420, 2003.
[45] S. Jin, X. Qu, C. Xu, D. Ma, and D. Wang, "An improved multi-value cellular automata model for heterogeneous bicycle traffic flow," Physics Letters A, vol. 379, no. 39, pp. 2409-2416, 2015.

[46] Y. Luo, B. Jia, J. Liu, W. H. K. Lam, X. Li, and Z. Gao, "Modeling the interactions between car and bicycle in heterogeneous traffic," Journal of Advanced Transportation, vol. 49, no. 1, pp. 29-47, 2015.

[47] Y. Liu, J. Guo, J. Taplin, and Y. Wang, "Characteristic analysis of mixed traffic flow of regular and autonomous vehicles using cellular automata," Journal of Advanced Transportation, vol. 2017, Article ID 8142074, 10 pages, 2017.

[48] S. E. Lee, E. C. B. Olsen, and W. W. Wierwille, A Comprehensive Examination of Naturalistic Lane-Changes, Publication Dot Hs Virginia Tech Transportation Institute, Blacksburg, VA, USA, 2004.

[49] T. G. Oketch, "New modeling approach for mixed-traffic streams with nonmotorized vehicles," Transportation Research Record, vol. 1705, no. 1, pp. 61-69, 2000.

[50] L. Feia, H. B. Zhua, and X. L. Hanb, "Analysis of traffic congestion induced by the work zone," Physica A: Statistical Mechanics and Its Applications, vol. 450, pp. 49-505, 2016.

[51] S.-W. Kim and W. Liu, "Cooperative autonomous driving: a Mirror neuron inspired intention awareness and cooperative perception approach," IEEE Intelligent Transportation Systems Magazine, vol. 8, no. 3, pp. 23-32, 2016. 